\title{
ÜBER EINE IN DER NEUEREN WERTVERTEILUNGSTHEORIE BETRACHTETE KLASSE TRANSZENDENTER FUNKTIONEN.
}

\author{
VON \\ LARS AHLFORS \\ in HeLsINGFors.
}

\section{Einleitung.}

In einer in diesem Heft S. 295-373 erscheinenden Arbeit, deren Hauptresultate zum Teil schon in den Abhandlungen aus dem mathematischen Seminar der Hamburgischen Universität» veröffentlicht worden sind, untersucht Herr RoLF Nhvanirina ${ }^{1}$ eingehend eine neue, wichtige Klasse von transzendenten, mehrdeutigen Funktionen $z(w)$, die durch folgende Eigenschaften ausgezeichnet sind:

I. $z(w)$ ist mit rationalem Charakter analytisch fortsetzbar in der ganzen Ebene, ausser möglicherweise, wenn man auf einen der endlichvielen Punkte $a_{1}, a_{2}, \ldots, a_{q}$ trifft.

2. $z(w)$ ist einwertig, d.h. den Mittelpunkten von verschiedenen Funktionselementen entsprechen immer verschiedene Funktionswerte.

3. Utber jedem Punkt $a_{i}$ liegt eine endliche Anzahl $\mu_{i}$ von logarithmischen Singularitäten. Ausserdem liegen über $a_{i}$ nur reguläre Elemente.

4. Die Riemannsche Fläche der Funktion $z(w)$, oder was dasselbe heisst, das von den Funktionswerten $z(w)$ gebildete Gebiet $G$ ist einfach zusammenhängend.

Herr Nevanlinna hat zunächst gezeigt, dass die zu diesen Funktionen gehörigen Riemannschen Flächen in gewisse Typen eingeteilt werden können, deren Struktur er vollständig beschreibt. Im Falle $q=2$ ist $z(w)$ eine lineare Transformation des Logarithmus, und auch im Falle $q=3, \mu_{1}=\mu_{2}=\mu_{3}=$ I gibt

\footnotetext{
- ${ }^{1}$ R. Nevanlinna: Über Riemannsche Flächen mit endlich vielen Windungspunkten.
} 
es, wenn von linearen Transformationen abgesehen wird, nur eine einzige Funktion mit den verlangten Eigenschaften. In allen übrigen Fällen existieren unendlich viele, wesentlich verschiedene Funktionen $z(w)$, die den Bedingungen des Problems genügen.

Ferner hat er bewiesen, dass das Wertgebiet der Funktionen $z(w)$ immer mit der in einem Punkt (z. B. im Unendlichen) punktierten Ebene identisch ist, sodass die Umkehrfunktion $w=w(z)$ eine überall im Endlichen definierte, meromorphe Funktion wird. Diese Funktion ist von der endlichen Ordnung $\frac{n}{2}$, wo $n=\sum_{1}^{q} \mu_{i}$ die Gesamtanzahl der Singularitäten von $w(z)$ bezeichnet, und besitzt aus dem Standpunkt der Wertverteilungstheorie besonders interessante Eigenschaften. Ihre einzigen defekten Werte sind nämlich die Werte $a_{i}$, und zwar sind die entsprechenden Defekte gleich $\frac{2 \mu_{i}}{n}$, sodass die Summe aller Defekte genau gleich 2 wird. Hierdurch ist es Herrn Nevandinna gelungen meromorphe Funktionen mit vorgeschriebenen, rationalen Defekten mit der Summe 2 zu konstruieren.

Um diese Resultate zu gewinnen, verwendet Herr Nevanuinna als hauptsächliches Hilfsmittel die asymptotische Integration einer Differentialgleichung, der die Funktion $w(z)$ genügt. Es ist nun sehr interessant, dass man auch ohne Benützung dieser Differentialgleichung, nur durch Methoden der konformen Abbildung, zu denselben Resultaten wie Herr Nevantinna kommen kann.

In der vorliegenden Arbeit wird die Untersuchung der Nevandinnaschen Funktionen auf die Untersuchung der konformen Abbildung von zwei schlichten Gebieten aufeinander zurückgeführt. Zur Lösung von Aufgaben dieser Art habe ich schon früher ${ }^{1}$ eine einfache Methode erfunden, die nur auf evidente Inhaltsund Längenabschätzungen sowie auf die Verwendung der Schwarzschen Ungleichung

$$
\left(\int f g d \alpha\right)^{2} \leqq \int f^{2} d \alpha \cdot \int g^{2} d \alpha
$$

beruht. Die Methode erweist sich auch in diesem Falle als erfolgreich und liefert, wie schon gesagt wurde, die wichtigsten von Nevandinna bewiesenen Resultate.

1 L. AHLFons: Untersuchungen zur Theorie der konformen Abbildung und der ganzen Funktionen (Acta Societatis Scient. Fennicae. Nova Series A. t. I. No. 9). 
Darüber hinaus beweise ich in dieser Arbeit nichts neues, aber die Verallgemeinerungsfähigkeit meiner Methode liegt an der Hand.

Aus Darstellungsgründen werde ich nicht sofort an den allgemeinsten Fall herangehen, sondern führe zunächst in aller Breite den Beweis für den einfachsten, nicht-trivialen Fall $n=q=3$ aus, und zeige dann wie die Ưberlègung für den allgemeinen Fall abgeändert werden muss. Im allgemeinen Fall treten nämlich gewisse unwesentliche, aber schwer umgehbare Komplikationen auf, die bei einer anderen Darstellungsweise leicht die einfache, zugrumdeliegende Idee des Beweises in den Hintergrund stellen würden.

\section{Der Fall $n=q=3$.}

I. Die Struktur der Riemannschen Fläche $\boldsymbol{F}$ der Funktion $z(w)$, welche den in der Einleitung gestellten Bedingungen mit $\mu_{1}=\mu_{2}=\mu_{3}=1$ genügt, geht

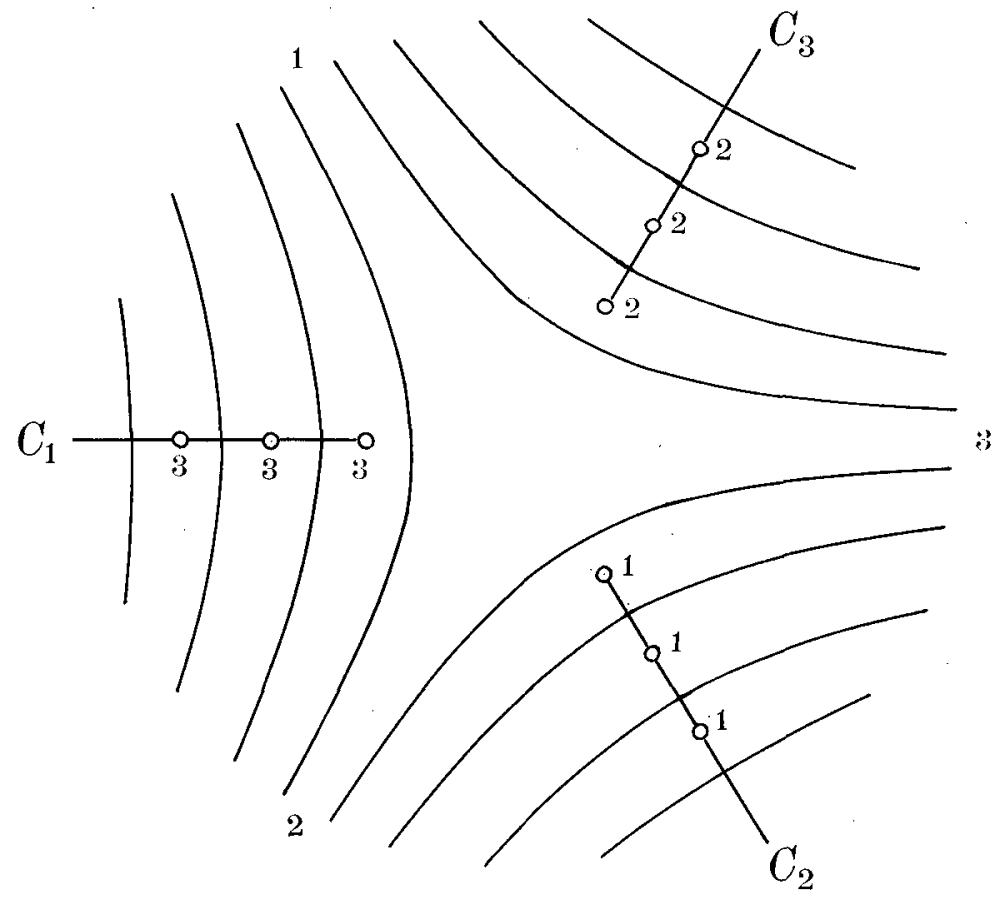

Fig. I.

nach Nevanlinna aus Fig. I hervor, die durch stetige Abbildung von $F$ auf eine schlichte Ebene gewonnen ist. Jedem Blatt der Fläche entspricht ein von den Kurven der Figur begrenztes Fundamentalgebiet. Das Kernpolygon mit den drei 48-31356. Acta mathematica. 58. Imprimé le 11 mars 1932. 
im Unendlichen gelegenen Ecken ist Bild eines Blattes, in dem alle die über den Punkten $a_{i}$ gelegenen Punkte singulär sind, während die anderen Fundamentalgebiete nur zwei unendlich ferne Randpunkte besitzen, und demnach zu Blättern mit nur zwei Singularitäten und einem über $a_{1}, a_{2}$ oder $a_{3}$ gelegenen regulären Punkt gehören.

Mit Nevanimana betrachten wir ausser $z(w)$ noch die linear polymorphe Funktion $\zeta=\zeta(w)$, welche die universelle Ủberlagerungsfläche der in $a_{1}, a_{2}, a_{3}$ punktierten $w$-Ebene auf den Kreis $|\zeta|<$ I konform abbildet. Diese Funktion ist natürlich nichts anderes als die Modulfunktion eines von $v$ linear abhängigen Argumentes. Thre verschiedenen Zweige gehen durch die linearen Transformationen einer Gruppe $S$ auseinander hervor, welche von den drei Fundamentalsubstitutionen $S_{1}, S_{2}$ und $S_{3}$ mit der identischen Relation $S_{1} S_{2} S_{3}=$ I erzeugt wird. $S_{i}$ ist diejenige Transformation, welche ein willkürlich gewählter Hauptzweig erleidet, wenn $w$ einen positiven Umlauf um $a_{i}$ ausführt.

Betrachtet man die Funktion $\zeta(w)$ auf der Riemannschen Fläche $F$, so ist sie immer noch mehrdentig, und ihre Zweige substituieren sich in einer Untergruppe von $S$. Um einen auf $F$ eindeutigen $Z_{w e i g} \bar{\zeta}(w)$ zu fixieren müssen wir die Fläche aufschneiden längs Schnitten, die durch sämtliche über $a_{1}, a_{2}$ und $a_{3}$ gelegene innere Punkte der Fläche hindurchgeht. Auf der so aufgeschnittenen Fläche ist $\zeta(w)$ überall regulär, und folglich jeder ihrer Zweige eine eindeutige Funktion.

Ein geeignetes Schnittsystem erhält man, wenn man in Fig. 1 die gleichbezeichneten Punkte durch Kurven verbindet, wie in der Figur gezeigt wird. Der Kurve $C_{1}$, die durch die mit 3 bezeichneten Punkte hindurchgeht, entspricht in der $w$-Ebene eine Kurve, die sich unendlich oft um $a_{2}$ im positiven und um $a_{1}$ im negativen Sinn herumwindet und bei jedem Umlauf durch $a_{3}$ hindurchgeht. Die Kurve kann so gewählt werden, dass die einzelnen Umläufe genau über einander liegen. Ausserdem können wir erreichen, dass diesen Umläufen in der -Ebene Kreisbogen entsprechen, die zum Einheitskreis orthogonal sind. In unserem Falle geschieht dieses so, dass man die Umläufe längs demjenigen durch $a_{3}$ gehenden Kreis unternimmt, in bezug auf welchen die Punkte $a_{1}$ und $a_{2}$ spiegelbildlich sind. Werden die Kurven $C_{2}$ und $C_{3}$ ähnlich gewählt, so erhält man in der $w$-Ebene eine Figur von dem in Fig. 2 gezeigten Aussehen.

Wir können annehmen, dass die logarithmischen Windungspunkte zu dem Hauptzweig von $\zeta(w)$ gehören, und dass diese Windungspunkte durch die $\mathbf{A b}$ - 
bildung auf die $\zeta$-Ebene in die Randpunkte $\varepsilon, \varepsilon^{2}$ und $\varepsilon^{3}\left(\varepsilon=e^{\frac{2 \pi i}{3}}\right)$ übergehen. Dann entspricht dem Anfangspunkt des Schnittes $C_{1}$ der Punkt $S_{2} \varepsilon^{3}=S_{1}^{-1} \varepsilon^{3}$. Die von diesem Punkt ausgehenden Orthogonalkreisbogen, die zu den Punkten $S_{2}^{\overline{2}} \varepsilon^{3}$ und $S_{1}^{-2} \varepsilon^{3}$ führen, entsprechen dem linken und rechten Ufer des ersten in $a_{3}$ beginnenden Umlaufes. Dem zweiten Umlauf gehören die zwischen $S_{2}^{2} \varepsilon^{3}$ und $S_{2}^{3} \varepsilon^{3}$ bzw. $S_{1}^{-2} \varepsilon^{3}$ und $S_{1}^{-3} \varepsilon^{3}$ verlaufenden Orthogonalkreise, usw. Die ganze Kurve $C_{1}$ wird also auf zwei aus einem Punkt ausgehende, gebrochene Kurvenzüge abgebildet, die aus den zwischen den Punkten $S_{2} \varepsilon^{\mathbf{3}}, S_{2}^{2} \varepsilon^{\mathbf{3}}, S_{2}^{3} \varepsilon^{\mathbf{3}}, \ldots$ und $S_{1}^{-1} \varepsilon^{3}, S_{1}^{-2} \varepsilon^{3}, S_{1}^{-3} \varepsilon^{3}, \ldots$ gezogenen Orthogonalkreisbogen bestehen.

Ebenso werden die Kurven $C_{2}$ und $C_{3}$ auf ähnliche Kurvenzüge abgebildet,

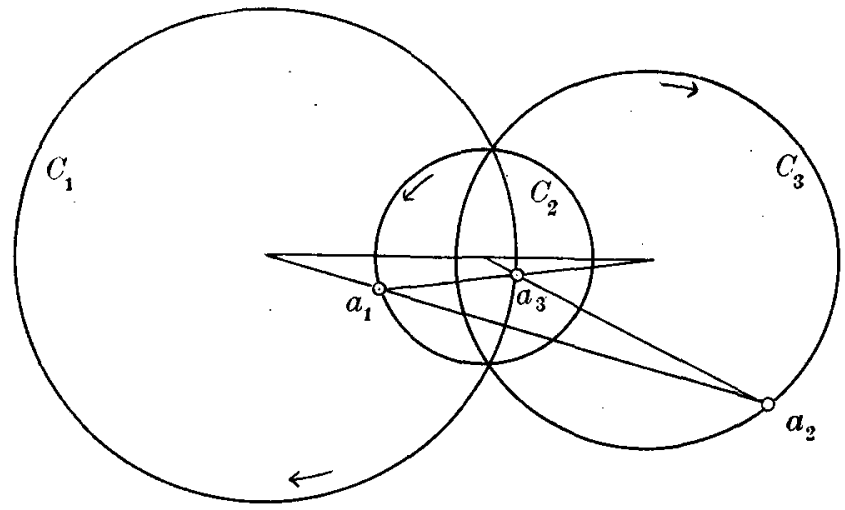

Fig. 2 .

welche die Punktfolgen $S_{3} \varepsilon, S_{3}^{2} \varepsilon, S_{3}^{3} \varepsilon, \ldots$ und $S_{2}^{-1} \varepsilon, S_{2}^{-2} \varepsilon, S_{2}^{-3} \varepsilon, \ldots$ bzw. $S_{1} \varepsilon^{2}$, $S_{1}^{2} \varepsilon^{2}, S_{1}^{3} \varepsilon^{2}, \ldots$ und $S_{3}^{-1} \varepsilon^{2}, S_{3}^{-2} \varepsilon^{2}, S_{3}^{-3} \varepsilon^{2}, \ldots$ verbinden.

Als Bild der aufgeschnittenen Fläche $F$ erhalten wir also ein aus unendlich vielen Orthogonalkreisdreiecken begrenztes Polygongebiet $Q$, dessen Ecken sich gegen die drei Punkte $\varepsilon, \varepsilon^{2}$ und $\varepsilon^{3}$ häufen (Fig. 3). Durch Identifizierung der zugeordneten Randpunkte, die zu demselben Punkte eines Schnittes von $F$ gehören, bekommt man ein ein-eindeutiges Bild der ursprünglichen Riemannschen Fläche.

In der $z$-Ebene erhält man das Bildgebiet der aufgeschnittenen Fläche $F$, indem man das Wertgebiet $G$ von $z(w)$ mit drei Einschnitten versieht, welche den Kurven $C_{1}, C_{2}$ und $C_{3}$ entsprechen. Das so entstandene Gebiet werde mit $G$ bezeichnet. Die zusammengesetzte Funktion $\zeta=\bar{\zeta}(w(z))$ vermittelt die konforme Abbildung von $\bar{G}$ auf das Kreisbogenpolygon $Q$ in der $\zeta$-Ebene. 
Die ganze Untersuchung der Funktion $w(z)$ ist nun auf die Untersuchung dieser konformen Abbildung zurückgeführt, denn die zwischen der $\zeta$-Ebene und der $w$-Ebene bestehende Zuordnung, welche durch die Modulfunktion bestimmt wird, beherrscht man vollständig. Eine Schwierigkeit bei der Behandlung der genannten Abbildung besteht darin, dass man den Verlauf der erwähnten Einschnitte nicht kennt. Stattdem weiss man aber, welche die einander zugeordneten Randpunkte

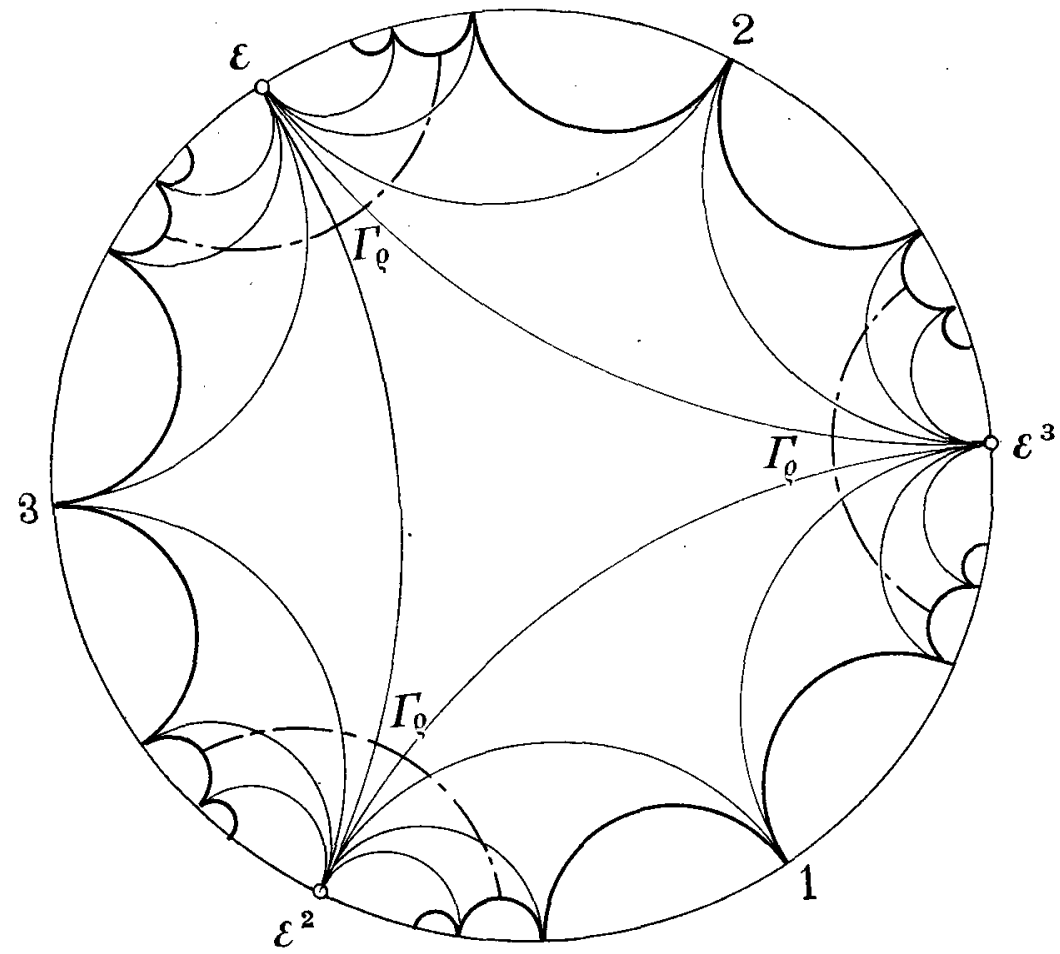

Fig. 3.

von $Q$ sind, die einem und demselben Punkt eines Einschnittes entsprechen, und es zeigt sich, dass diese Kenntnis für unsere Zwecke ausreicht.

2. Bildet man mit Hilfe der Funktionen

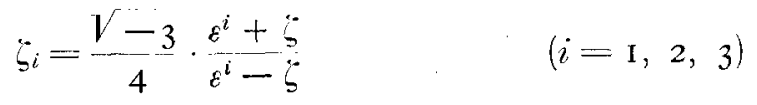

den Kreis $|\zeta|<$ I auf die obere Halbebene ab, wobei nacheinander die Punkte $\varepsilon, \varepsilon^{2}$ und $\varepsilon^{3}$ in den Punkt $\infty$ übergeführt werden, so erhält man als Bild des Polygons $Q$ ein Gebiet, das von Halbkreisen mit auf der reellen Achse gelegenen 
Mittelpunkten begrenzt wird. In der $\zeta_{1}$-Ebene entsprechen den Punkten $\varepsilon^{3}$ und $\varepsilon^{2}$ die Punkte $\pm \frac{\mathrm{I}}{4}$. Die Kreisbogen, welche sich gegen den Punkt $\varepsilon$ häufen, also die Bilder des rechten Ufers von $C_{1}^{\prime}$ und des linken Ufers von $C_{3}$, werden auf kongruente Halbkreise vom Radius ${ }_{2}$ mit den Mittelpunkten $\pm\left(n+\frac{\mathrm{I}}{4}\right)$, $n=\mathrm{I}, 2,3, \ldots$ abgebildet (Fig. 4). In den $\zeta_{2^{-}}$und $\zeta_{3}$-Ebenen erhält man zufolge der Symmetrie genau dieselbe Figur.

In jeder der $\zeta_{i}$-Ebenen wird nun ein Kreis um den Nullpunkt mit dem Radius $\varrho \geqq \frac{3}{4}$ geschlagen und der zum Bildgebiet von $Q$ gehörige Bogen $T_{e}^{i}$ dieses Kreises bestimmt. In der $\zeta$-Ebene entsprechen diesen Bogen drei kongruente Ortho-

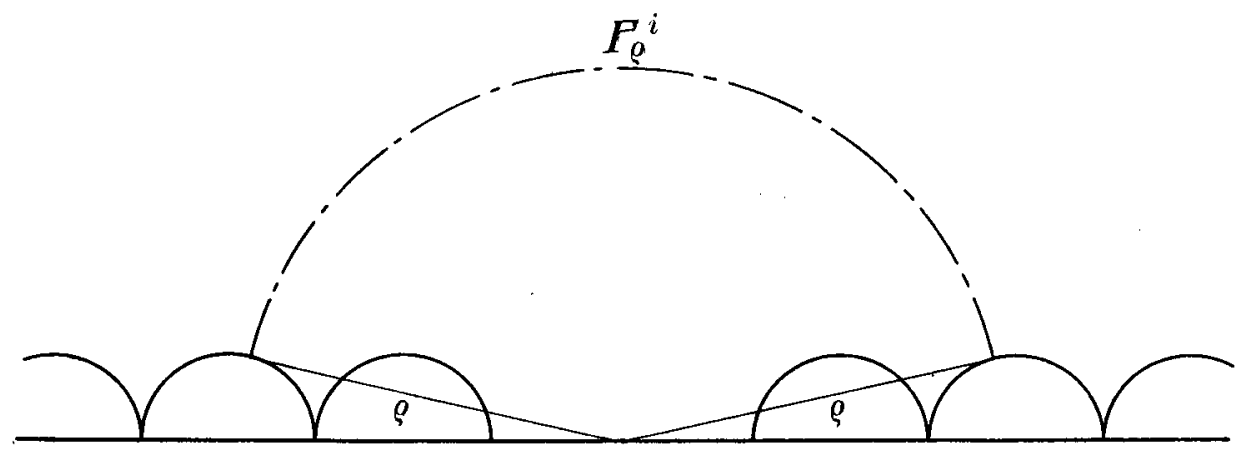

Fig. 4.

gonalkreisbogen, welche einander nicht schneiden und in bezug auf die Durchmesser durch $\varepsilon, \varepsilon^{2}$ und $\varepsilon^{3}$ symmetrisch liegen. Die zyklisch aufeinanderfolgenden Endpunkte dieser Bogen sind zugeordnete Randpunkte von $Q$, d.h. wenn man die zugeordneten Punkte identifiziert, so bilden die drei Bogen zusammen eine geschlossene Kurve, die wir mit $I_{Q}$ bezeichnen. In der $w$-Ebene entspricht der Kurve $I_{\ell}^{\prime}$ eine auf der Riemannschen Fläche $I^{\prime}$ geschlossene, sich selbst nicht schneidende Kurve, und in der z-Ebene erhält man als Bild eine einfache Kurve, die den Bildpunkt von $\zeta=0$ umschlingt. Offenbar können wir ohne weiteres voraussetzen, dass dies der Punkt $z=0$ ist.

3. Die Anzahl der in der $z$-Ebene innerhalb der Bildkurve von $I_{\varrho}$ gelegenen Wurzel der Gleichung $w(z)=a$ bezeichnen wir mit $v(\varrho, a)$. Man erhält sie auch, wenn man die Anzahl der verschiedenen, innerhalb $\Gamma_{Q}$ gelegenen Werte von $\zeta(a)$ berechnet. 
Um diese Anzahl zu bestimmen, betrachte ich in der $\zeta$-Ebene die Moduldreiecke mit den Spitzen I, $\varepsilon$, - I und I, $\varepsilon^{2}$, - I. Diese Dreiecke bilden zusammen ein Fundamentalgebiet der Umkehrfunktion von $\zeta(w)$. Für jedes a gibt es also einen Wert $\zeta(a)$, der zu einem dieser Dreiecke gehört, und falls $a \neq a_{1}$ und $a_{2}$ ist, so wird dieser Wert für ein genügend grosses $g$ von der Kurve $I_{c}$ eingeschlossen. Ausserdem können zu dem von $\Gamma_{\ell}$ eingeschlossenen Gebiet noch gewisse Transformierte $S_{1}^{-1} \zeta, S_{1}^{-2} \zeta, \ldots$ oder $S_{2} \zeta, S_{2}^{2} \zeta, \ldots$ des betrachteten Wertes gehören, jenachdem der Ausgangswert zum ersten oder zweiten Dreieck gehört. Die Anzahl dieser äquivalenten Punkte erhält man, wenn man im ersten Falle zur $\zeta_{1}$-Ebene und im zweiten Falle zur $\zeta_{2}$-Ebene übergeht. Dort entspricht den äquivalenten Punkten eine Folge von Werten, die durch eine Translation um eine Einheit in der Richtung der reellen Achse auseinander hervorgehen. Im ersten Falle sind alle Bildpunkte links und im zweiten Falle rechts von der imaginären Achse gelegen. Die Anzahl dieser Punkte, die innerhalb $\Gamma_{\ell}^{\prime \prime}$ bzw. $\Gamma_{e}^{*}$ gelegen sind, ist offenbar gleich $\varrho+O(\mathrm{I})$, wo mit $O(\mathrm{I})$ ein beschränktes Glied bezeichnet ist.

Dieselbe U̇berlegung kann dann wiederholt werden, erst mit den Moduldreiecken $\varepsilon, \varepsilon^{2},-\varepsilon$ und $\varepsilon, \varepsilon^{3},-\varepsilon$, dann mit den Dreiecken $\varepsilon^{2}, \varepsilon^{3},-\varepsilon^{2}$ und $\varepsilon^{2}$, $\varepsilon,-\varepsilon^{2}$. Jedesmal erhält man dieselbe Anzahl, $\varrho+O(\mathrm{I})$, von neuen, innerhalb $I_{\varrho}$ gelegenen Bildpunkten, wenn nur im ersten Falle $a \neq a_{2}, a_{3}$ und im zweiten Falle $a \neq a_{1}, a_{3}$ ist. Addiert man alle so gefundenen Bildpunkte, so wird also

$$
\left\{\begin{array}{l}
v(\varrho, a)=3 \varrho+O(\mathrm{I}) \text { für } a \neq a_{1}, a_{2}, a_{3}, \\
v\left(\varrho, a_{i}\right)=\varrho+O(\mathrm{I}),
\end{array} \quad(i=\mathrm{I}, 2,3) .\right.
$$

Wir haben hiermit bewiesen, dass die Funktion $w(z)$ alle Werte $a \neq a_{1}, a_{2}$, $a_{3}$ asymptotisch gleich oft annimmt in den von den Bildkurven der Kurven $I_{0}$ begrenzten Gebieten, während die Anzahl der $a_{i}$-Stellen asymptotisch kleiner ist. Der Defekt, d.h. die Anzahl der fehlenden $a_{i}$-Stellen im Verhältnis zur normalen Anzahl, beträgt für jedes $a_{i}$

$$
\bar{\delta}\left(a_{i}\right)=\lim _{\varrho \rightarrow \infty} \frac{\nu(\varrho, a)-v\left(\varrho, a_{i}\right)}{\nu(\varrho, a)}=\frac{2}{3}
$$

Die Summe der Defekte ist $3 \cdot \frac{2}{3}=2$.

Unser Ziel ist zu beweisen, dass diese Defekte nicht nur auftreten, wenn die $z$-Ebene in der obigen, von den Kurven $I_{\varrho}$ bestimmten Weise erschöpft wird, 
sondern auch wenn man die Ebene durch konzentrische Kreise ausschöpft, wie es die Nevandinnasche Wertverteilungstheorie fordert. Um dies zu zeigen, muss man beweisen, dass die Bildkurven der Kurven $\Gamma_{\varrho}$ annähernde Kreisform haben, d. h. dass das Verhältnis zwischen dem grössten und dem kleinsten Abstand eines Punktes auf der Bildkurve von $\Gamma_{q}$ zum Punkte $z=0$ den Grenzwert I hat. Dieser Beweis bildet die Hauptschwierigkeit der ganzen Untersuchung.

4. Die Umkehrfunktion der Funktion $\zeta=\bar{\zeta}(w(z))$ sei im folgenden mit $z=\bar{z}(\zeta)$ bezeichnet. Wie schon bemerkt wurde, wird $\Gamma_{Q}$ durch Vermittlung von $\bar{z}(\zeta)$ auf eine geschlossene Kurve der $z$-Ebene abgebildet, welche den Nullpunkt umgibt. Auf dieser Kurve sei $r_{1}(\varrho)$ der kleinste und $r_{2}(\varrho)$ der grösste Wert von $|z|$. Ausserdem wird die logarithmische Schwankung $\omega(\varrho)=\log r_{2}(\varrho)-\log r_{1}(\varrho)$ eingeführt.

Mit $s=\log z$ bezeichnen wir denjenigen Zweig des Logarithmus, dessen Imaginarteil zwischen o und $2 \pi$ liegt. Das Bild von $\Gamma_{\varphi}$ in der $s$-Ebene ist eine Kurve, welche zwei Punkte mit den Ordinaten o und $2 \pi$ verbindet. Ihre Projektion auf der reellen Achse ist gleich $\omega(\varrho)$. Man überzengt sich lejcht, dass die Länge einer solchen Kurve wenigstens gleich der Diagonale eines Rechtecks mit den Seiten $2 \pi$ und $\omega(\varrho)$ sein muss, und erhält demnach die Ungleichung

$$
\sum_{i=1}^{3} \int_{I^{i}}\left|\frac{d s}{d \zeta_{i}}\right|\left|d \zeta_{i}\right| \geqq \sqrt{4 \pi^{2}+\omega(\varrho)^{2}}
$$

Durch Anwendung der Schwarzschen Ungleichung bekommt man hieraus

$$
4 \pi^{2}+\omega(\varrho)^{2} \leqq \sum_{1}^{3} \int_{I_{\ell}^{i}}\left|d \zeta_{i}\right| \cdot \sum_{1}^{3} \int_{I_{\varrho}^{i}}\left|\frac{d s}{d \zeta_{i}}\right|^{2}\left|d \zeta_{i}\right|
$$

und, da die Länge jeder Kurve $\Gamma_{Q}^{i}$ höchstens gleich $\pi \varrho$ ist,

$$
4 \pi^{2}+\omega(\varrho)^{2} \leqq 3 \pi \varrho \cdot \sum_{1}^{3} \int_{\Gamma_{\ell}^{i}}\left|\frac{d s}{d \zeta_{i}}\right|^{2}\left|d \zeta_{i}\right|
$$

Dividiert man diese Ungleichung durch $3 \pi \varrho$ und integriert in bezug auf $\varrho$ zwischen den Grenzen $\varrho^{\prime}$ und $\varrho^{\prime \prime}\left(3 \leqq \varrho^{\prime}<\varrho^{\prime \prime}\right)$, so wird 
( 1 )

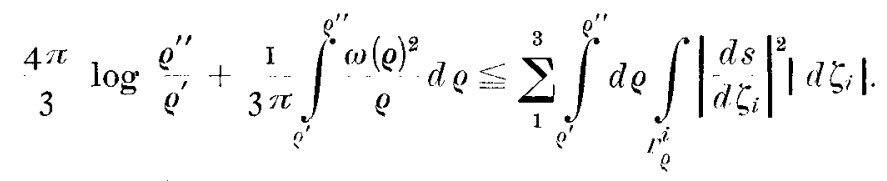

Der rechtsstehende Ausdruck stellt den Inhalt des Flächenstïcks dar, das durch die Bildkurven von $\Gamma_{q^{\prime}}$ und $\Gamma_{Q^{\prime \prime}}$ von dem Parallelstreifen $O \leqq I(s) \leqq 2 \pi$ abgeschnitten wird. Diese Fläche ist höchstens gleich $2 \pi\left(\log r_{2}\left(\varrho^{\prime \prime}\right)-\log r_{1}\left(\varrho^{\prime}\right)\right)=$ $=2 \pi\left(\log \frac{r_{1}\left(\varrho^{\prime \prime}\right)}{r_{1}\left(\varrho^{\prime}\right)}+\omega\left(\varrho^{\prime \prime}\right)\right)$. Benützt man diese Abschätzung, so folgt aus (1)

$$
\frac{2}{3} \log \frac{\varrho^{\prime \prime}}{\varrho^{\prime}}-\log \frac{r_{1}\left(\varrho^{\prime \prime}\right)}{r_{1}\left(\varrho^{\prime}\right)} \leqq \omega\left(\varrho^{\prime \prime}\right)-\frac{\mathrm{I}}{6 \pi^{2}} \int_{\varrho^{\prime}}^{\varrho^{\prime \prime}} \frac{\omega(\varrho)^{2}}{\varrho} d \varrho .
$$

Aus dieser Ungleichung ergibt sich schon, dass $\lim _{\ell \rightarrow \infty} r_{1}(\varrho)=\infty$ ist, d. h. dass das Wertgebiet $G$ mit der ganzen $z$-Ebene identisch ist, denn der auf der rechten Seite von (2) stehende Ausdruck ist im allgemeinen beschränkt. Schreibt man nämlich

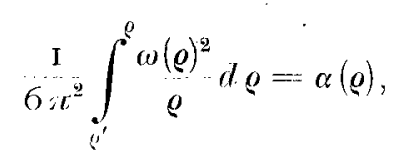

und ist

$$
\omega(\varrho)>a(\varrho)+\mathrm{I}
$$

so folgt

oder

$$
(\alpha(\varrho)+\mathrm{I})^{2}<6 x^{2} \frac{\rho \alpha(\varrho)}{d \varrho}
$$

$$
\frac{d \varrho}{\varrho}<6 \pi^{2} \frac{d \alpha(\varrho)}{(1+\alpha(\varrho))^{2}}
$$

Wenn (3) in einem ganzen Intervall $A$ gilt, so folgt

$$
\int_{i} \frac{d \varrho}{\varrho}<6 \pi^{2} \int_{i} \frac{d \alpha(\varrho)}{(\mathrm{I}+\alpha(\varrho))^{2}} \leqq 6 \pi \tau^{2} \int_{\alpha=0}^{\infty} \frac{d \alpha}{(\mathrm{I}+\alpha)^{2}}=6 \pi^{2}
$$

Also kann sich kein Intervall $A$ ins Unendliche strecken, d. h. es gibt beliebig grosse Werte $\varrho$, für welche (3) nicht gilt. Setzt man für $\varrho^{\prime \prime}$ einen solchen Wert 
ein, so folgt aus (2)

$$
\log \frac{r_{1}\left(\varrho^{\prime \prime}\right)}{r_{1}\left(\varrho^{\prime}\right)} \geqq \frac{2}{3} \log \frac{\varrho^{\prime \prime}}{\varrho^{\prime}}-1
$$

Da $r_{1}(\varrho)$ eine wachsende Funktion von $\varrho$ ist, geht hieraus die Behauptung $\lim _{\ell \rightarrow \infty} r_{1}(\varrho)=\infty$ unmittelbar hervor.

5. Nachdem wir bewiesen haben, dass das Wertgebiet der Funktion $z(w)$ die ganze $z$-Ebene ist, können wir uns denken, dass die bisher nur schematisch aufgefasste Figur I die wirkliche Zerlegung der $z$-Ebene darstellt, und dass die Kurven $C_{i}$ die Bilder der paarweise zugeordneten Randstiicke von $Q$ sind.

Wenn $r$ genügend gross ist $\left(r \geqq r_{2}\left(\frac{3}{4}\right)\right)$ trifft der Kreis $|z|=r$ alle drei Kurven $C_{i}$. Derjenige Bogen dieses Kreises, der einen Punkt auf dem linken Ufer von $C_{1}$ mit einem Punkt auf dem rechten Ufer von $C_{2}$ verbindet, heisse $\theta_{r}^{(1)}{ }^{1}$ Den ähnlich bestimmten Bogen zwischen $C_{2}$ und $C_{3}$ bezeichnen wir mit $\boldsymbol{\theta}_{r}^{(2)}$ und den zwischen $C_{3}$ und $C_{1}$ gelegenen Bogen mit $\boldsymbol{\theta}_{r}^{(3)}$. Der zum Bogen $\boldsymbol{\theta}_{r}^{(i)}$ gehörige Zentriwinkel sei $\theta_{i}(r)$. Dann ist $\sum_{i=1}^{3} \theta_{i}(r) \leqq 2 \pi$.

Dem Bogen $\theta_{r}^{(i)}$ entspricht in der $\zeta_{i}$-Ebene ein Querschnitt des Bildgebiets von $Q$, der den Punkt $\frac{\sqrt{2}}{2}$ vom unendlich fernen Punkt trennt. Daraus ist ersichtlich, dass die Variation von arg $\left(\zeta_{i}-\frac{\sqrt{-\mathrm{I}}}{2}\right)$ auf $\theta_{r}^{(i)}$ wenigstens gleich $x$ ist.

Geht man zur $\sigma_{1}=\log \left(\zeta_{i}-\frac{V-I}{2}\right)$-Ebene über, wobei der Zweig des Logarithmus so festgelegt wird, dass sein Imaginarteil zwischen $-\frac{\pi}{2}$ und $\frac{3 \pi}{2} \operatorname{liegt}$, so erhält man also als Bild von $\theta_{r}^{(i)}$ eine Kurve, welche wenigstens die Länge $\pi$ hat. Es gilt somit

$$
\int_{\theta_{r}^{(i)}}\left|\frac{d \sigma_{i}}{d z}\right||d z| \geqq \pi
$$

woraus mit Hilfe der Schwarzschen Ungleichung folgt

${ }^{1}$ Falls mehrere solche Bogen vorhanden sind, sei $\theta_{r}^{(1)}$ irgendeiner unter diesen.

49-31356. Acta mathematica. 58. Imprimé le 11 marg 1932. 


$$
x^{2} \leqq \int_{\theta_{r}^{(i)}}|d z| \int_{\theta_{r}^{(i)}}\left|\frac{d \sigma_{i}}{d z}\right|^{2}|d z|=v \theta_{i}(r) \int_{\theta_{r}^{(i)}}\left|\frac{d \sigma_{i}}{d z}\right|^{2}|d z|
$$

Diese Ungleichung dividieren wir durch $r \theta_{i}(r)$ und integrieren in bezug auf $r$ zwischen den Grenzen $r_{2}\left(\varrho^{\prime}\right)$ und $r_{1}\left(\varrho^{\prime \prime}\right)$. Man erhält dann

$$
\pi^{2} \int_{r_{2}\left(\rho^{\prime}\right)}^{r_{1}\left(\rho^{\prime \prime}\right)} \frac{d r}{r \theta_{i}(r)} \leqq \int_{r_{2}\left(\varrho^{\prime}\right)}^{r_{1}\left(\rho^{\prime \prime}\right)} d r \int_{\theta_{r}^{(i)}}\left|\frac{d \sigma_{i}}{d z}\right|^{2}|d z|
$$

Auf der rechten Seite von (4) steht wieder der Inhalt einer gewissen Fläche. Diese Fläche liegt erstens - wenn $\sigma_{i}=\xi+i \eta$ geschrieben wird - zwischen den Geraden $\xi=\log \left(\varrho^{\prime}-\frac{\mathrm{I}}{2}\right)$ und $\xi=\log \left(\varrho^{\prime \prime}+\frac{\mathrm{I}}{2}\right) \cdot{ }^{1}$ Andererseits ist sie im Bildgebiet der oberen $\zeta_{i}$-Halbebene erhalten, d. h. sie liegt zwischen den zwei Kurven

$$
\left\{\begin{array}{l}
\eta=-\arcsin \frac{e^{-\xi}}{2} \\
\eta=\pi+\arcsin e_{2}^{-\xi}
\end{array}\right.
$$

Also ist die ganze Fläche höchstens gleich

$$
\pi \log \frac{\varrho^{\prime \prime}+\frac{2}{2}}{\varrho^{\prime}-\frac{1}{2}}+2 \int_{\log \left(\varrho^{\prime}-\frac{1}{2}\right)}^{\log \left(\varrho^{\prime \prime}+\frac{1}{2}\right)} \arcsin \frac{e^{-\xi}}{2} d \xi=x \log {\frac{\varrho^{\prime \prime}}{\varrho^{\prime}}}^{\prime}+O\left(\frac{\mathrm{I}}{\varrho^{\prime}}\right)
$$

Trägt man dies in (4) ein, so bekommt man schliesslich

$$
\pi \int_{r_{2}\left(\varrho^{\prime}\right)}^{r_{1}\left(\varrho^{\prime \prime}\right)} \frac{d r}{r \theta_{i}(r)} \leqq \log \frac{\varrho^{\prime \prime}}{\varrho^{\prime}}+O\left(\frac{\mathrm{I}}{\varrho^{\prime}}\right), \quad(i=\mathrm{I}, 2,3)
$$

Diese drei Ungleichungen addieren wir, und benutzen, dass nach dem Satz vom arithmetischen und harmonischen Mittel

${ }^{1}$ Es wird $\varrho^{\prime}>\frac{3}{4}$ angenommen. 


$$
\sum_{1}^{: 3} \theta_{i}(r) \gtreqless \sum_{1}^{3} \theta_{i}(v) \gtreqless \underset{2 \pi}{9}
$$

ist. Man erhïlt so die Ungleichung

oder

$$
{ }_{2}^{3} \log \frac{r_{1}\left(\varrho^{\prime \prime}\right)}{r_{2}\left(\varrho^{\prime}\right)} \leqq \log \frac{\varrho^{\prime \prime}}{\varrho^{\prime}}+O\left(\begin{array}{l}
\mathrm{I} \\
\varrho^{\prime}
\end{array}\right)
$$

$$
{ }_{3}^{2} \log \frac{\varrho^{\prime \prime}}{\varrho^{\prime}-\log r_{1}\left(\varrho^{\prime \prime}\right)}{ }_{r_{1}\left(\varrho^{\prime}\right)} \geqq-\omega\left(\varrho^{\prime \prime}\right)+O\left(\begin{array}{l}
1 \\
\varrho^{\prime}
\end{array}\right) .
$$

Die zwei Fundamentalungleichungen (2) und (5) ergeben zusammen

$$
\omega\left(\varrho^{\prime}\right)+\omega\left(\varrho^{\prime \prime}\right)=\sigma \frac{1}{6 \pi} \int_{\varrho^{\prime}}^{\varrho^{\prime \prime}} \omega(\varrho)^{2} d \varrho+o\left(\begin{array}{l}
1 \\
\varrho
\end{array}\right)
$$

eine Ungleichung, welche also immer besteht, wenn ${ }_{4}^{3}<\varrho^{\prime}<\varrho^{\prime \prime}$. Wir werden zeigen, dass diese Ungleichung $\lim _{\rho \rightarrow \infty} \omega(\varrho)=0$ und sogar eine etwas schärfere Beziehung zur Folge hat.

6. Wir wählen ein festes $\varrho>{ }_{16}^{9}$ und wenden die Ungleichung (6) an auf ein $\varrho^{\prime}$ aus dem Intervall $V \varrho \leqq \varrho^{\prime} \leqq \varrho$ und ein $\varrho^{\prime \prime}$ aus dem Intervall $\varrho \leqq \varrho^{\prime \prime} \leqq \varrho V \varrho$. Die Ungleichung bringen wir auf die Form

$$
\begin{aligned}
& \text { (7) } \omega\left(\varrho^{\prime}\right)+\omega\left(\varrho^{\prime \prime}\right) \leqq\left(2 \omega\left(\varrho^{\prime \prime}\right)-\frac{\mathrm{I}}{6 u^{\prime \prime}} \int_{\varrho^{\prime \prime}}^{\varrho^{\prime \prime}(\varrho)^{2}} d \varrho\right) \\
& +\left(2 \omega\left(\varrho^{\prime}\right)-\cdots \frac{1}{6 x^{2}} \int_{\varrho^{\prime}}^{{ }^{\prime} \omega(\varrho)^{2}}-d \varrho\right)+O\left(\begin{array}{c}
\mathrm{I} \\
\varrho^{\prime}
\end{array}\right) .
\end{aligned}
$$

Wir versuchen jetzt $\varrho$ " innerhalb des Intervalls $(\varrho, \varrho l \varrho)$ so zu wählen, dass der erste Klammerausdruck in (7) sein Minimum erreicht. Dieses Minimum bezeichnen wir mit $\delta$. Es gilt folglich, wenn wieder

$$
\sigma \pi \tau^{2} \int_{!}^{1} \varrho_{\varrho}^{\prime \prime} \omega(\varrho)^{2} d \varrho-\alpha\left(\varrho^{\prime \prime}\right)
$$


gesetzt wird,

$$
2 \omega\left(\varrho^{\prime \prime}\right) \geqq \alpha\left(\varrho^{\prime \prime}\right)+\delta
$$

oder

$$
\left(\alpha\left(\varrho^{\prime \prime}\right)+\delta\right)^{2} \leqq 24 x^{2} \frac{\varrho^{\prime \prime} d \alpha\left(\varrho^{\prime \prime}\right)}{d \varrho^{\prime \prime}}
$$

für $\varrho \leqq \varrho^{\prime \prime} \leqq \varrho \sqrt{\varrho .}$ Hieraus folgt

$$
\frac{d \varrho^{\prime \prime}}{\varrho^{\prime \prime}} \leqq 24 \pi t^{2} \frac{d \alpha\left(\varrho^{\prime \prime}\right)}{\left(\alpha\left(\varrho^{\prime \prime}\right)+\delta\right)^{2}}
$$

und durch Integration über das ganze Intervall $(\varrho, \varrho \sqrt{\varrho})$

$$
\frac{\mathrm{I}}{2} \log \varrho \leqq 24 \pi^{2} \int_{\varrho}^{\varrho^{V} \varrho} \frac{d \alpha\left(\varrho^{\prime \prime}\right)}{\left(\alpha\left(\varrho^{\prime \prime}\right)+\delta\right)^{2}} \leqq 24 \pi^{2} \int_{\alpha=0}^{\infty} \frac{d \alpha}{(\alpha+\delta)^{2}}=\frac{24 \pi^{2}}{\delta}
$$

oder

$$
\delta \leqq \frac{48 \pi^{2}}{\log \varrho}
$$

In derselben Weise wird gezeigt, dass auch das Minimum des zweiten Klammerausdrucks in $(7)$ im Intervall $v \varrho \leqq \varrho^{\prime} \leqq \varrho$ kleiner als oder gleich $\frac{4^{8} \pi^{\sharp}}{\log \varrho}$ ist. Man kann also $\varrho^{\prime}$ und $\varrho^{\prime \prime}$ aus den vorgeschriebenen Intervallen wählen, sodass nach $(7)$

$$
\omega\left(\varrho^{\prime}\right)+\omega\left(\varrho^{\prime \prime}\right) \leqq \frac{9^{6} \pi^{2}}{\log \varrho}+O\left(\frac{\mathbf{I}}{\varrho^{\prime}}\right)
$$

wird.

Wir nehmen dann eine Zahl $\eta$, die grösser als $\omega\left(\varrho^{\prime}\right)$ und $\omega\left(\varrho^{\prime \prime}\right)$ ist, und bezeichnen mit $\Delta$ das logarithmische Mass ${ }^{1}$ der Menge der zwischen $\varrho^{\prime}$ und $\varrho^{\prime \prime}$ liegenden Werte $\varrho$, für welche $\omega(\varrho) \geqq \eta$ ist. Dann folgt aus (6) in Verbindung mit (8)

$$
\frac{\eta^{2}}{6 \pi^{2}} d \leqq \frac{96 \pi \pi^{2}}{\log \varrho}+O\left(\frac{\mathrm{I}}{\sqrt{\varrho}}\right)
$$

oder

$$
\Delta \leqq \frac{576 \pi^{4}}{\eta^{2} \log \varrho}+O\left(\frac{\mathrm{I}}{\sqrt{\varrho}}\right)
$$

${ }^{1}$ d. h. das über diese Menge ausgestreckte Integral $\int \frac{d \varrho}{\rho}$. 
Da nun $\omega(\varrho)$ nach Voraussetzung nicht in dem ganzen geschlossenen Intervall $\left(\varrho^{\prime}, \varrho^{\prime \prime}\right)$ grösser als $\eta$ ist, so kann man in diesem Intervall zwei so nahe an $\varrho$ gelegene Werte $\varrho_{1} \leqq \varrho$ und $\varrho_{2} \geqq \varrho$ finden, für welche $\omega\left(\varrho_{1}\right)$ und $\omega\left(\varrho_{2}\right) \leqq \eta$ ist, dass

$$
\log \frac{\varrho_{2}}{\varrho_{1}} \leqq \frac{576 \pi^{4}}{\eta^{2} \log \varrho}+O\left(\frac{\mathrm{I}}{\sqrt{\varrho}}\right)
$$

Ferner ist

$$
\omega(\varrho) \leqq \log r_{2}\left(\varrho_{2}\right)-\log r_{1}\left(\varrho_{1}\right)=\log \frac{r_{1}\left(\varrho_{2}\right)}{r_{1}\left(\varrho_{1}\right)}+\omega\left(\varrho_{2}\right),
$$

woraus nach (5) folgt

$$
\omega(\varrho) \leqq \frac{2}{3} \log \frac{\varrho_{g}}{\varrho_{1}}+\omega\left(\varrho_{1}\right)+\omega\left(\varrho_{2}\right)+O\left(\frac{\mathrm{I}}{\varrho_{1}}\right) \leqq \frac{384 x^{4}}{\eta^{2} \log \varrho}+2 \eta+O\left(\frac{\mathrm{I}}{\sqrt{\varrho}}\right) .
$$

Die rechtsstehende Schranke wird zu einem Minimum für

$$
\eta=\sqrt[3]{ }_{\frac{384 \pi^{4}}{\log \varrho}}
$$

und bei dieser Wahl ist die Bedingung $\eta>\omega\left(\varrho^{\prime}\right)$ und $\omega\left(\varrho^{\prime \prime}\right)$ wegen (8) erfüllt, wenn $\varrho$ genügend gross ist. Man erhält so die gewünschte Abschätzung

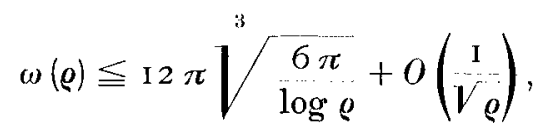

welche zeigt, dass $\omega(\varrho)$ mit wachsendem $\varrho$ tatsächlich gegen Null strebt wie $\frac{\mathrm{I}}{\sqrt[3]{\log \varrho}}$.

7. Aus (2) und (5) folgt nunmehr unmittelbar, dass

$$
\log \frac{r_{1}\left(\varrho^{\prime \prime}\right)}{r_{1}\left(\varrho^{\prime}\right)}-\frac{2}{3} \log \frac{\varrho^{\prime \prime}}{\varrho^{\prime}}=O\left(\frac{1}{\sqrt[3]{\log \varrho^{\prime}}}\right) .
$$

Man schliesst hieraus auf die Existenz des Grenzwertes

$$
\lim _{\rho \rightarrow \infty}\left(\log r_{1}(\varrho)-\frac{2}{3} \log \varrho\right)=\log A
$$


und erhält die asymptotische Darstellung

$$
r_{1}(\varrho)=A \varrho^{\frac{2}{3}}\left(\mathrm{I}+O\left(\begin{array}{c}
\mathrm{I} \\
3^{3} \\
\sqrt{\log \varrho}
\end{array}\right)\right)
$$

Ebenso gilt

$$
r_{2}(\varrho)=A \varrho^{\frac{2}{3}}\left(I+O\left(\frac{I}{\sqrt[3]{\log \varrho}}\right)\right)
$$

Die Wertverteilungsgrössen $n(r, a)$ erhält man mit Hilfe der Resultate in Nr. 3. Es ist offenbar

$$
n\left(r_{1}(\varrho), a\right) \leqq \nu(\varrho, a) \leqq n\left(r_{2}(\varrho), a\right)
$$

Wählt man zunächst $\varrho$ sodass $r_{1}(\varrho)=r$, woraus folgt

$$
\varrho=A^{\prime} r^{\cdot 2}\left(\mathrm{I}+O\left(\frac{\mathrm{I}}{\sqrt[3]{\log r}}\right)\right), A^{\prime}=A^{-\ddot{2},}
$$

so wird (S. 382)

$$
\begin{aligned}
& n(r, a) \leqq \nu(\varrho, a)=3 A^{\prime} r^{\cdot 3}\left(\mathrm{I}+O\left(\frac{\mathrm{I}}{\sqrt[3]{\log r}}\right)\right) \text { für } a \neq a_{1}, a_{2}, a_{3} \\
& n\left(r, a_{i} \leqq\right.
\end{aligned}
$$

Wird $\varrho$ andererseits so gewählt, dass $r_{2}(\varrho)=r$, so erhält man in derselben Weise die umgekehrten Ungleichungen. Es gilt mithin

$$
\begin{aligned}
& n(r, a)=3 A^{\prime} r^{\frac{3}{2}}\left(\mathrm{I}+O\left(\frac{\mathrm{I}}{\sqrt[3]{\log r}}\right)\right) \text { für } a \neq a_{1}, a_{2}, a_{3} \\
& n\left(r, a_{i}\right)=A^{\prime} r^{2}\left(\mathrm{I}+O\left(\begin{array}{c}
\mathrm{I} \\
\sqrt[3]{\log r}
\end{array}\right)\right), \quad(i=1,2,3) .
\end{aligned}
$$

Wenn $n(r)$ das Maximum von $n(r, a)$ für alle Werte von $a$ bezeichnet, so gilt auch 


$$
\lim _{r \rightarrow \infty} \frac{n(r)}{r^{3 / 3}}=A^{\prime}
$$

d. h. die Funktion $w(z)$ ist vom mittleren Typus der Ordnung ${ }_{2}^{3}$. Ferner ist

$$
\begin{aligned}
& \lim _{r \rightarrow \infty} \frac{n(r, a)}{n(r)}=\mathrm{I} \text { für } a \neq a_{1}, a_{2}, a_{3} \\
& \lim _{r \rightarrow \infty} \frac{n\left(r, a_{i}\right)}{n(r)}=\frac{\mathrm{I}}{3} .
\end{aligned}
$$

Die einzigen Werte mit einem positiven Defekt sind also die Werte $a_{i}$ und der Defekt jedes Wertes beträgt

$$
\delta\left(a_{i}\right)=\lim _{r \rightarrow \infty}\left(\mathrm{x}-\frac{n\left(r, a_{i}\right)}{n(r)}\right)=\frac{2}{3}
$$

wobei die Defekte jetzt im ursprünglichen NEvaniannaschen Sinn berechnet sind. ${ }^{1}$

Wir fassen noch die Resultate dieses Abschnittes zusammen:

Die, bis auflineare Transformationen, einzige in einem einfach zusammenhängenden Gebiet definierte, eindeutige Funktion ohne multiplen Stellen, deren Umkehrfunktion mur über den Stellen $a_{1}, a_{2}, a_{3}$ je eine transzcndente Singularität besitzt, ist eine in der ganzen Ebene meromorphe Funktion vom mittleren Typus der Ordnung $\frac{3}{2}$ mit den drei Defekten $\delta\left(a_{1}\right)=\delta\left(a_{2}\right)=\delta\left(a_{3}\right)=\frac{2}{3}$. Die Summe der. Defekte ist also genun gleich 2 .

\section{Der allgemeine Fall.}

A. 8. Wir untersuchen dann den Fall, wo die Funktion $z(w)$ über jedem der Punkte $a_{1}, a_{2}, \ldots, a_{q}(q>3)$ eine und nur eine logarithmische Singularitait besitzt. Ausserdem nehmen wir zunächst an, dass alle Singularitäten in demselben Blatt liegen, d.h. dass man ein Anfangselement finden kann, von dem aus alle Singularitäten längs solchen Wegen erreichbar sind, die sich selbst und

1 Eigentlich sind die Nevanlinnaschen Defekte nicht mit Hilfe der Funktionen $n(r, a)$, sondern mit Hilfe der damit verwandten Funktionen $N(r, a)$ definiert. Es ist aber leicht zu sehen, dass ein durch $n(r, a)$ definierter Defekt einen gleich grossen, durch $N(r, a)$ gemessenen Defekt ergibt, wogegen das umgekehrte nicht immer gilt. 
einander nicht schneiden. Die Punkte $a_{1}, \ldots, a_{q}$ seien so numeriert, dass die entsprechenden Wege zyklisch im positiven Drehungssinn aufeinanderfolgen. Die schematische Figur 5, welche die Struktur der Riemannschen Fläche veranschaulicht, entsteht genau wie die entsprechende Figur I mit dem Unterschied, dass das erste Fundamentalgebiet jetzt $q$ im Unendlichen gelegene Ecken hat.

Von der Funktion $\zeta=\zeta\left(w ; a_{1}, \ldots, a_{q}\right)$, welche die universelle Überlagerungsfläche der in $a_{1}, \ldots, a_{q}$ punktierten $w$-Ebene auf den Einheitskreis abbildet,

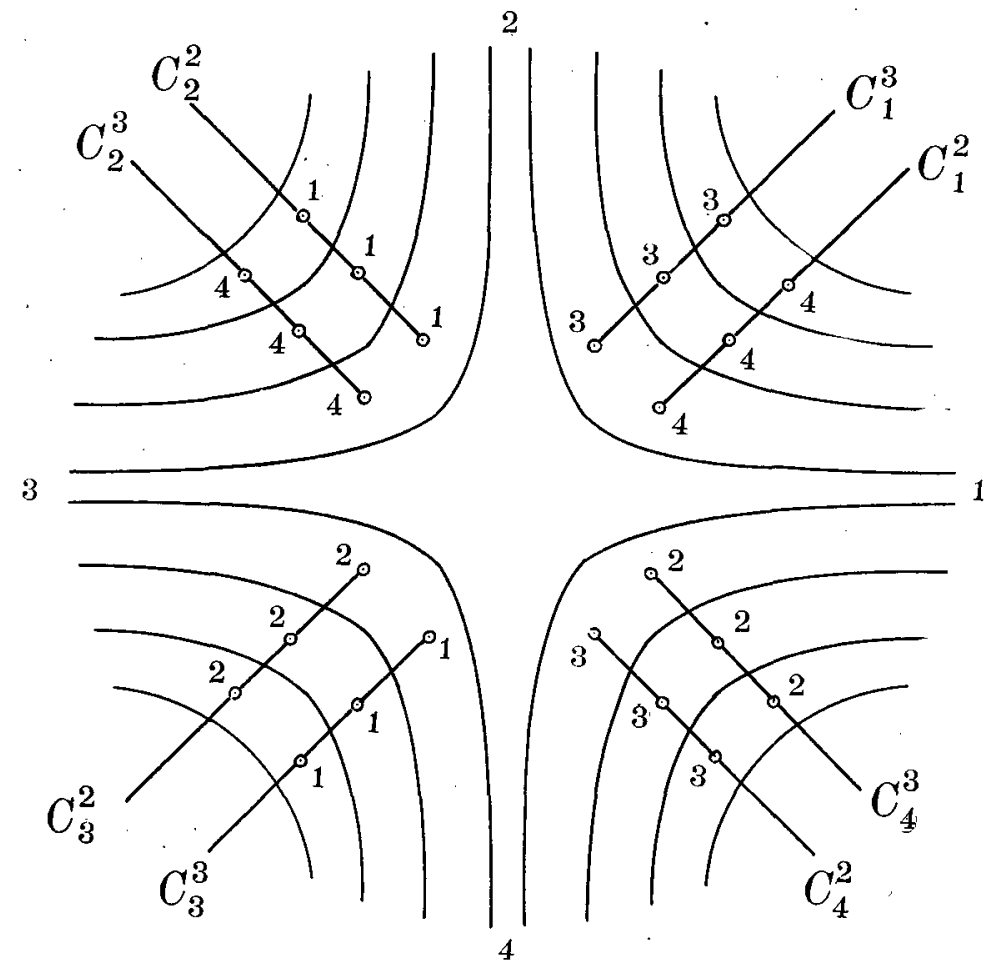

Fig. 5.

soll wieder ein eindeutiger Zweirg $\zeta=\zeta(w)$ fixiert werden. Dazu wird ein Schnittsystem eingeführt, das in Fig. 5 von den Kurven $C_{i}^{k}(i=\mathrm{I}, \ldots, q ; k=2, \ldots$, $q-\mathrm{I})$ repräsentiert wird. $C_{i}^{k}$ ist eine Kurve, welche in dem an die Seite $(i, i+1)$ des Grundpolygons grenzenden »logarithmischen Ende»verläuft und durch die dort gelegenen Bildpunkte von $a_{i-k+1}$ hindurchgeht. $^{1}$ In jedem zum betreffenden logarithmischen Ende gehörigen Fundamentalgebiet soll der Schnitt $C_{i}^{k}$ die Singularität $a_{i}$ und die vorhergehenden Schnitte $C_{i}^{k-1}, C_{i}^{k-2}, \ldots, C_{i}^{2}$ von der

1 Hier und im folgenden sollen alle Indizes auf ihre Reste modulo $q$ rednziert werden. 
Singularität $a_{i+1}$ und den nachfolgenden Schnitten $C_{i}^{k+1}, C_{i}^{k+2}, \ldots, C_{i}^{k-1}$ trennen. Dann gehört zu $C_{i}^{k}$ in der $w$-Ebene eine Kurve, die sich unendlich oft um $a_{i}, a_{i-1}, \ldots, a_{i-k+2}$ im negativen und um $a_{i+1}, a_{i+2}, \ldots, a_{i+q-k}$ im positiven Sinn herumwindet und bei jedem Umlauf durch $a_{i-k+1}$ hindurchgeht. Man kann es wieder so einrichten, dass alle zu demselben Schnitt gehörigen Umläufe genau

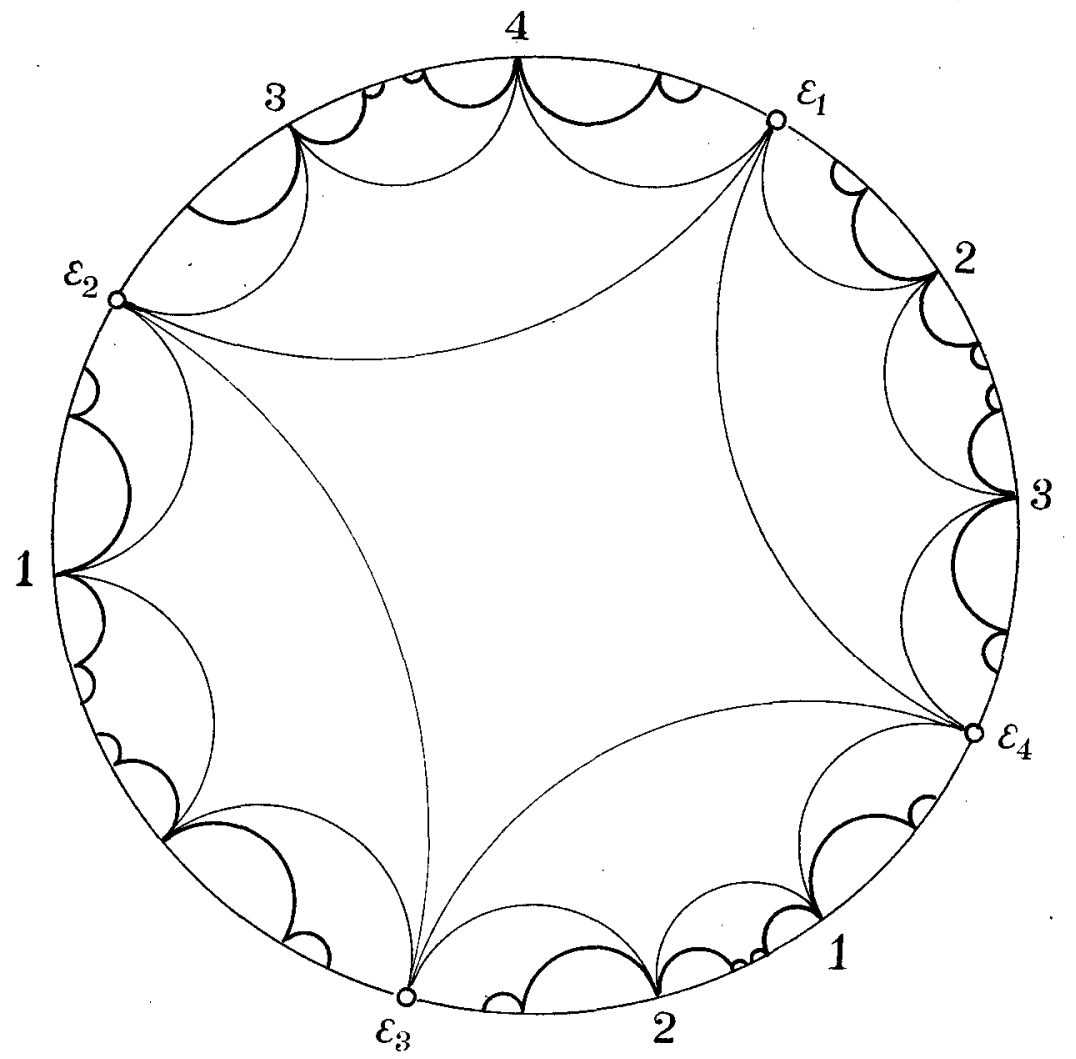

Fig. 6.

übereinander liegen, und dass jedem Umlauf in der $\zeta$-Ebene ein Orthogonalkreisbogen des Einheitskreises entspricht.

Den Singularitäten von $z(w)$ entspreche in der $\zeta$-Ebene durch Vermittlung des Hauptzweiges von $\zeta(w)$ die Punkte $\varepsilon_{1}, \ldots, \varepsilon_{q}$ auf der Peripherie des Einheitskreises, und bei einem positiven Umlauf um $a_{i}$ soll dieser Zweig der Transformation $S_{i}$ unterworfen werden. Dann entspricht dem Anfangspunkt des Schnittes $C_{i}^{k}$ der Punkt $T_{i, k}^{\prime} \varepsilon_{i-k+1}=T_{i, k}^{\prime \prime} \varepsilon_{i-k+1}$, wo $T_{i, k}^{\prime}=S_{i+1} S_{i+2} \ldots S_{i+q-k}$ und $T_{i, k}^{\prime \prime}=S_{i}^{-1} S_{i-1}^{-1} \ldots S_{i-k+2}^{-1}$. Als Bild des ersten zu $C_{i}^{k}$ gehörigen Umlaufes erhält 50-31356. Acta mathematica. 58. Imprimé lo 12 mars 1932. 
man die von dem genannten Punkt ausgehenden Orthogonalkreisbogen, welche in den Punkten $T_{i, k}^{\prime 2} \varepsilon_{i-k+1}$ und $T_{i, k}^{\prime \prime 2} \varepsilon_{i-k+1}$ endigen. Allgemein gehört zu dem linken Ufer des $p$ :ten Umlaufes der Orthogonalkreisbogen zwischen den Punkten $T_{i, k}^{\prime p_{1}} \quad \varepsilon_{--k+1}$ und $T_{i, k}^{\prime p+1} \varepsilon_{i-k+1}$, während zu dem rechten Ufer der Kreisbogen zwischen $T_{i, k}^{\prime \prime p} \varepsilon_{i-k+1}$ und $T_{i, k}^{\prime \prime p+1} \varepsilon_{i-k+1}$ gehört. Die beiden Ufer werden also auf Kreisbogenzüge abgebildet, die gegen die anziehenden Fixpunkte $\omega_{i, k}^{\prime}$ und $\omega_{i, k}^{\prime \prime}$ der Transformationen $T_{i, k}^{\prime}$ und $T_{i, k}^{\prime \prime}$ konvergieren.

$T_{i, 2}^{\prime \prime}$ und $T_{i, q-1}^{\prime}$ sind parabolisch mit den einzigen Fixpunkten $\varepsilon_{i}$ und $\varepsilon_{i+1}$. Ferner ist für $k=2, \ldots, q-2 \quad T_{i, k}^{\prime}=T_{i, k+1}^{\prime \prime}$, sodass auch $\omega_{i, k}^{\prime}=\omega_{i, k+1}^{\prime \prime}$. Also ist die ganze Anzahl der infragekommenden Fixpunkte gleich $q(q-2)$, d. h. die

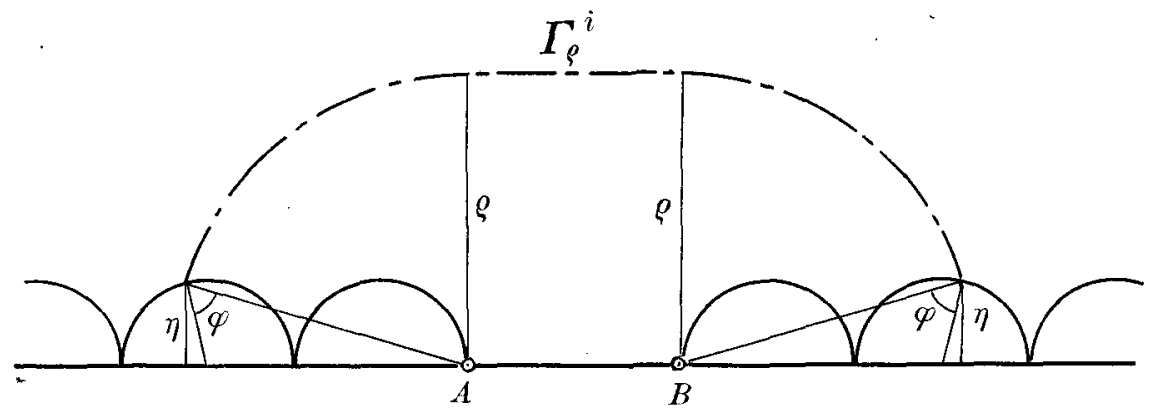

Fig. 7 .

Riemannsche Fläche $F$ wird auf ein Kreispolygon $Q$ mit paarweise zugeordneten Seiten abgebildet, dessen Ecken sich gegen $q(q-2)$ Punkte auf der Peripherie des Einheitskreises häufen (siehe Fig. 6, wo $q=4$ ).

9. Man betrachte wieder eine Abbildung des Einheitskreises auf die obere $\zeta_{i}$-Halbebene $(i=\mathrm{I}, \ldots, q)$, welche den Punkt $\varepsilon_{i}$ in den unendlich fernen Punkt überführt. Die Abbildung soll so normiert sein, dass der Transformation $S_{i}$ eine Translation um eine Einheit in der Richtung der positiven reellen Achse entspricht. Als Bild derjenigen Seiten von $Q$, die sich gegen $\varepsilon_{i}$ häufen, erhält man dann wie in Fig. 4 zwei von den Bildpunkten $A$ und $B$ der Anfangspunkte der Schnitte $C_{i}^{2}$ und $C_{i-1}^{q-1}$ ausgehende Folgen aneinandergelegter, kongruenter Halbkreise vom Radius $\frac{\mathrm{I}}{2}$ (Fig. 7).

Wir definieren dann die Querschnitte $\Gamma_{e}^{i}$ in fast derselben Weise wie in Nr. 2. Man schlage um $A$ und $B$ Halbkreise vom Radius g, nehme von beiden Halbkreisen nur die äusseren Quadranten und verbinde ihre senkrecht über $A$ 
und $B$ gelegenen Endpunkte mit einer zur reellen Achse parallelen Strecke. Der zum Bildgebiet von $Q$ gehörige Teil dieser zusammengesetzten Linie sei $I_{Q}^{i}$.

Durch diese Definition ist aber noch nicht erreicht, dass der linke Endpunkt von $\Gamma_{e}^{i}$ dem rechten Endpunkt von $\Gamma_{\varrho}^{i+1}$ zugeordnet ist. Um zu einer geschlossenen Kurve' $\Gamma_{\varrho}$ zu kommen, müssen also noch gewisse Kurvenstücke hinzugefügt werden.

Dazu bilden wir den Einheitskreis noch auf die obere $\zeta_{i}^{k}$-Halbebene $(k=2$, $\ldots, q-2)$ ab, sodass der Fixpunkt $\omega_{i, k}^{\prime}=\omega_{i, k+1}^{\prime \prime}$ in den Punkt $\infty$ übergeht. Dann entspricht der Transformation $I_{i, k}^{\prime \prime} \equiv T_{i, k+1}^{\prime \prime}$ eine Ähnlichkeitstransformation vom Bildpunkt des zweiten Fixpunktes aus (als Nullpunkt gewählt) mit dem Proportionalitätsfaktor $\lambda>\mathrm{I}$. Als Bild der Seiten von $Q$, die sich gegen $\omega_{i, k}^{\prime}$

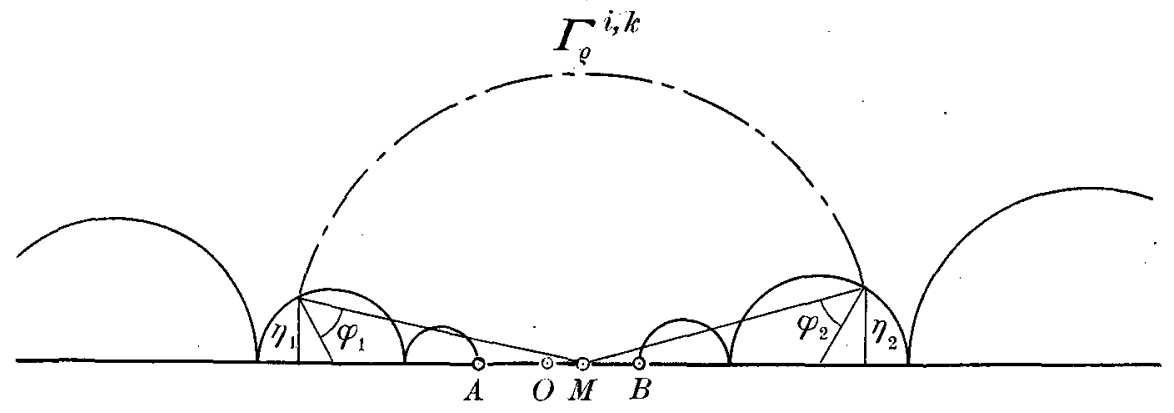

Fig. 8 .

häufen, erhält man beiderseits von $O$ eine Folge perspektivisch gelegener Halbkreise (Fig. 8).

In der $\zeta_{i}^{2}$-Ebene betrachten wir dann den Halbkreis über die Strecke $A B$ zwischen den Bildpunkten der Anfangspunkte von $C_{i}^{2}$ und $C_{i}^{s}$, und vergrössern ihn homothetisch vom Punkte $O$ aus bis er durch den zugeordneten Punkt des linken Endpunktes von $T_{e}^{i}$ hindurchgeht. Den zum Bildgebiet von $Q$ gehörigen Teil dieses Kreises nennen wir $\Gamma_{\varrho}^{i, 2}$. Allgemein soll das in der $\zeta_{i}^{k}$ Ebene $(k=2$, $\ldots, q-3)$ gelegene Kurvenstück $\Gamma_{\varrho}^{i, k}$ durch den zugeordneten Punkt des linken Endpunktes von $\Gamma_{e}^{i, k-1}$ hindurchgehen und auf einem homothetischen Kreis zu dem Orthogonalkreis durch die Transformierten der Anfangspunkte von $C_{i}^{k}$ und $C_{i}^{k+1}$ liegen. Nur das letzte Kurvenstück, $\Gamma_{\varrho}^{i, q-2}$, muss etwas anders definiert werden, nämlich als der Orthogonalkreisbogen, der in der $\zeta_{i}^{q-2}$-Ebene die zugeordneten Punkte des linken Endpunktes von $\Gamma_{\ell}^{i, q-3}$ und des rechten Endpunktes 
von $\Gamma_{\ell}^{i+1}$ verbindet. Alsdann entspricht der Kurve $\Gamma_{Q}$ in der $\zeta$-Ebene, welche aus

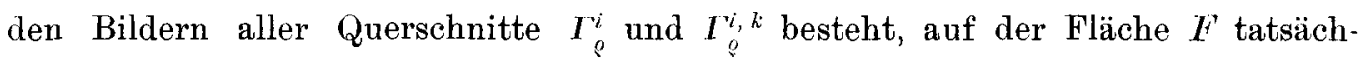
lich eine geschlosssene Kurve.

Io. Es sei wieder $v(\varrho, a)$ die Anzahl der von $I_{\varphi}$ eingeschlossenen Stellen, denen in der $w$-Ebene ein über. a gelegener Punkt entspricht. Diese Anzahl kann in folgender. Weise bestimmt werden.

In der $\zeta$ Ebene bildet das Orthogonalkreispolygon, dessen Ecken $\varepsilon_{i+1}, \varepsilon_{i+2}$, $\ldots, \varepsilon_{i+q}=\varepsilon_{i}$ und die Bildpunkte der Anfangspunkte der Kurven $C_{i}^{2}, C_{i}^{3}, \ldots$, $C_{i}^{q-1}$ sind, ein vollständiges Fundamentalgebiet der Funktion $\zeta(w)$. In diesem Gebiet liegt also sicher ein Wert von $\zeta(a)$, und wenn $a \neq a_{i}$ und $a_{i+1}$ ist, so wird dieser Wert für genügend grosse $\varrho$ von der Kurve $\Gamma_{\ell}$ eingeschlossen. Ausserdem schliesst aber $\Gamma_{\ell}$ noch eine Folge von äquivalenten Bildpunkten dieses Wertes ein, welche, jenachdem in welchem Teil des Fundamentalgebiets der betrachtete Wert $\zeta(a)$ liegt, durch positive Potenzen einer der Transformationen $T_{i, 2}^{\prime}, T_{i, 2}^{\prime \prime}=T_{i, 3}^{\prime}, T_{i, 3}^{\prime \prime}=T_{i, 4}^{\prime}, \ldots, T_{i, q-2}^{\prime \prime}=T_{i, q-1}^{\prime}, T_{i, q-1}^{\prime \prime}$ gegeben sind.

Für die parabolischen Substitutionen $T_{i, 2}^{\prime}=S_{i}^{-1}$ und $T_{i, 4-1}^{\prime \prime}=S_{i+1}^{\prime}$ berechnet man sofort die Anzahl dieser äquivalenten Punkte zu $\varrho+O(\mathrm{I})$, wie in Nr. 3 . Aber auch für die hyperbolischen Transformationen erhält man asymptotisch dieselbe Anzahl, denn zwischen den Querschnitten $I_{n}^{i, k}$ und $\Gamma_{n+1}^{i, k}$ liegt, wenn $n$ eine ganze Zahl ist, genau ein Bildpunkt.

Da dieselbe U̇berlegung für $i=1,2, \ldots, q$ wiederholt werden kann, so folgt, wenn $a$ von allen $a_{i}$ verschieden ist, dass die gesamte Anzahl der von $I_{\%}$ eingeschlossenen Werte $a$ gleich

$$
\nu(\varrho, a)=q \varrho+O(\mathrm{I})
$$

ist, während

$$
v\left(\varrho, a_{i}\right)=(q-2) \varrho+O(\mathrm{I}), \quad(i=\mathrm{I}, \ldots, q)
$$

Alle Werte werden also asymptotisch gleich oft angenommen mit Ausnahme der Werte $a_{i}$, welche den Defekt

$$
\tilde{\delta}\left(a_{i}\right)=\lim _{\rho \rightarrow \infty} \frac{\nu(\varrho, a)-\nu\left(\varrho, a_{i}\right)}{\nu(\varrho, a)}=\frac{2}{q}
$$

besitzen. Die Summe aller Defekte ist also wieder gleich $q \cdot \frac{2}{q}==2$. Es soll aber noch wie im Falle $q=3$ gezeigt werden, dass diese Defekte wirkliche Defekte im Sinne von Nevanlinna sind. 
I I. Der Kurve $r_{0}$ entspricht in der $z$-Ebene eine Kurve, die den Nullpunkt umschlingt. Gehen wir zur $s=\log z$-Ebene über, so wird also in derselben Weise wie in $\mathrm{Nr} .4$

$$
\int_{\Gamma_{Q}}|d s|=\sum_{1}^{q} \int_{I_{Q}^{i}}\left|\begin{array}{l}
d s \\
d \zeta_{i}
\end{array}\right|\left|d \zeta_{i}\right|+\sum_{i}^{q} \sum_{2}^{q-1} \int_{I_{\ell}^{i, k}}\left|\frac{d s}{d \zeta_{i}^{k}}\right|\left|d \zeta_{i}^{k}\right| \geqq V_{4} \pi^{2}+\omega(\varrho)^{2}
$$

Wir wenden wieder die Schwarzsche Ungleichung $\left(\int f g d \alpha\right)^{2} \leqq \int f^{2} d \alpha \cdot \int g^{2} d \alpha$ an, indem wir in den Integralen der ersten Summe $f=\mathrm{I}, g=\left|\frac{d s}{d \zeta_{i}}\right|$ und in den

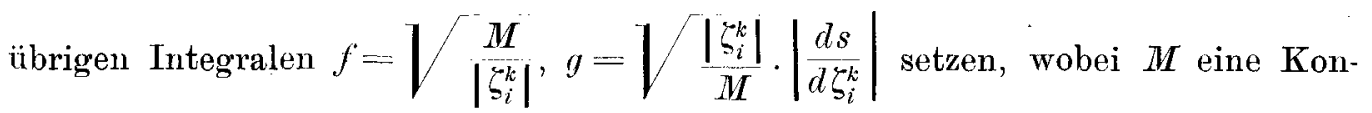
stante ist, deren Wert später bestimmt werden soll. Man erhält so

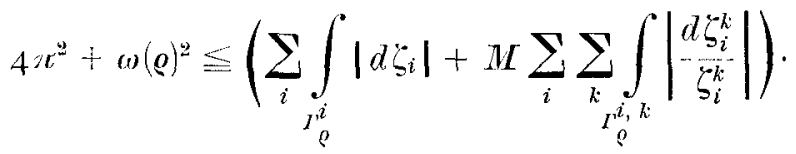

$$
\begin{aligned}
& \cdot\left(\sum_{i} \int_{I_{0}^{i}}\left|\frac{d s}{d \zeta_{i}}\right|^{2}\left|d \zeta_{i}\right|+\underset{M}{\mathrm{I}} \sum_{i} \sum_{k} \int_{I_{Q}^{i, k}}\left|\frac{d s}{d \zeta_{i}^{k}}\right|^{2}\left|\zeta_{i}^{k}\right|\left|d \zeta_{i}^{-k}\right|\right) .
\end{aligned}
$$

Alsdann beachten wir, dass

$$
\int_{r^{i}}\left|d \zeta_{i}\right| \leqq \pi \varphi+O(\mathrm{I})
$$

und

$$
\int_{\Gamma_{\rho}^{i, k}}\left|\begin{array}{c}
d \zeta_{i}^{k} \\
\zeta_{i}^{k}
\end{array}\right|=O(\mathrm{I})
$$

Dann wird, wenn $K$ eine genügend grosse Konstante ist,

$$
\frac{4 \pi^{2}+\omega(\varrho)^{2}}{\pi q(\varrho+K)} \leqq \sum_{i} \int_{\Gamma_{\varrho}^{i}}\left|\frac{d s}{d \zeta_{i}}\right|^{2}\left|d \zeta_{i}\right|+\sum_{M} \sum_{i} \sum_{k} \int_{\Gamma_{\ell}^{i, k}}\left|\frac{d s}{d \zeta_{i}^{k}}\right|^{2}\left|\zeta_{i}^{k}\right|\left|d \zeta_{i}^{k}\right|
$$


Die letzte Ungleichung wird nun wie in Nr. 4 integriert in bezug auf $\varrho$ zwischen den Grenzen $\varrho^{\prime}$ und $\varrho^{\prime \prime}$. Auf der rechten Seite erhält man dann Doppelintegrale von der Form

$$
\int_{\varrho^{\prime}}^{\varrho^{\prime \prime}} d \varrho \int_{I_{\rho}^{i}}\left|\frac{d s}{d \zeta_{i}}\right|^{2}\left|d \zeta_{i}\right|
$$

und

$$
\frac{\mathrm{I}}{M} \int_{\rho^{\prime}}^{g^{\prime \prime}} d \varrho \int_{r_{\rho}^{i, k}}\left|\frac{d s}{d \zeta_{i}^{\bar{k}}}\right|^{2}\left|\zeta_{i}^{k}\right|\left|d \zeta_{i}^{k}\right|
$$

Die geometrische Bedeutung jener ist klar: da die benachbarten Kurven $\Gamma_{\varrho}$ und $\Gamma_{\varrho}+d \varrho$ überall im Abstand $d \varrho$ von einander laufen, so stellt $d \varrho\left|d \zeta_{i}\right|$ das Flächenelement dar, und das Integral repräsentiert den Inhalt der von den Bildkurven der Kurven $\Gamma_{\varrho}^{i}$ erzeugten Fläche. Diese dagegen können nicht geometrisch interpretiert werden. Wir können aber zeigen, dass sie kleiner sind als die entsprechenden Flächeninhalte.

Dazu haben wir den kleinsten Abstand zwischen den Kurven $\Gamma_{\ell}^{i, k}$ und $\Gamma_{\varrho+d \rho}^{i, k}$ abzuschätzen. Dieser kleinste Abstand gehört notwendig zu einem Endpunkt des Querschnitts $\Gamma_{e}^{i, k}$. Es genügt daher, dass wir den genannten Abstand für alle Querschnittsendpunkte bestimmen.

In Fig. 8 sei $O A=a_{1}, O B=a_{2}$ und $M$ der Mittelpunkt des Kreises $\Gamma_{\ell}^{i, k}$. Die Abstände der Kreise $\Gamma_{\rho}^{i, k}$ und $\Gamma_{\varrho+d \rho}^{i, k}$ an den beiden Endpunkten des Querschnitts nennen wir $d n_{1}$ und $d n_{2}$. In den Fällen $k=\mathbf{I}, \ldots, q-4$, wo die Kreise homothetisch sind, gilt $d n_{1}: d n_{2}=a_{1}: a_{2}$.

Wenn wir die von den Querschnittsendpunkten beschriebenen Wege mit $\left|d \zeta_{i}^{k}\right|_{1}$ und $\left|d \zeta_{i}^{*}\right|_{2}$ bezeichnen, so gilt $d n_{1}=\left|d \zeta_{i}^{k}\right|_{1} \sin \varphi_{1}, d n_{2}{ }^{\circ}=\left|d \zeta_{i}^{*}\right|_{2} \sin \varphi_{2}$, wobei $\varphi_{1}$ und $\varphi_{2}$ die Winkel sind, unter welchen $\Gamma_{e}^{i, k}$ die von ihm getroffenen Halbkreise schneidet. Bezeichnet man nun die Ordinaten der Querschnittsendpunkte mit $\eta_{1}$ und $\eta_{2}$ und den Querschnittsradius mit $R$, und schreibt man statt $\lambda_{i}^{k}$ einfacher $\lambda$, so erhält man durch leichte Rechnung

$$
\sin \varphi_{1}=\frac{\eta_{1}}{R} \cdot \frac{\lambda-\mathrm{I}}{\lambda+\mathrm{I}}, \quad \sin \varphi_{2}=\frac{\eta_{2}}{R} \cdot \frac{\lambda-\mathrm{I}}{\lambda+\mathrm{I}}
$$


Es wird also

$$
\begin{aligned}
& d n_{1}=\left(\frac{\eta_{1}}{R}\right)^{2} \cdot \frac{\lambda-\mathrm{I}}{\lambda+\mathrm{I}} R \frac{\left|d \zeta_{i}^{k}\right|_{1}}{\eta_{1}} \\
& d n_{2}=\left(\frac{\eta_{2}}{R}\right)^{2} \cdot \frac{\lambda-\mathrm{I}}{\lambda+\mathrm{I}} R \frac{\left|d \zeta_{i}^{k}\right|_{\mathrm{g}}}{\eta_{2}}
\end{aligned}
$$

Ebenso folgt aus Fig. 8

$$
d \varrho=\left|d \zeta_{i}\right| \sin \varphi \leqq \frac{2(\varrho+\mathrm{I})}{\varrho} \eta\left|d \zeta_{i}\right| \leqq 4 \eta^{2} \cdot \frac{\left|d \zeta_{i}\right|}{\eta}
$$

wenn man $\varrho \geqq I$ voraussetzt. Da nun die zugeordneten Randpunkte in den $\zeta_{i^{-}}$ und $\zeta_{i}^{2}$-Ebenen durch lineare Transformationen ineinander übergehen, so gilt $\frac{\left|d \zeta_{i}\right|}{\eta}=\frac{\left|d \zeta_{i}^{2}\right|_{1}}{\eta_{1}}$ und folglich ist in der $\zeta_{i}^{*}$-Ebene

$$
d n_{1}=\left(\frac{\eta_{1}}{R}\right)^{2} \frac{\lambda-\mathrm{I}}{\lambda+\mathrm{I}} R \frac{\left|d \zeta_{i}\right|}{\eta} \geqq \frac{\mathrm{I}}{4}\left(\frac{\eta_{1}}{R \eta}\right)^{2} \frac{\lambda-\mathrm{I}}{\lambda+\mathrm{I}} R d \varrho .
$$

Der Ausdruck $\frac{\eta_{1}}{R \eta}$ nimmt für alle zugeordneten Halbkreispaare dieselben Werte an; es ist leicht zu sehen, dass sein Minimum positiv ist. Daher ist es klar, dass für genügend grosses $M$ die Ungleichung

$$
d n_{1}>\frac{R d \varrho}{M}
$$

für alle Werte $\varrho$ gilt. Ferner liegt auf dem ganzen Bogen $\Gamma_{\varrho}^{i, 2}$ der Quotient $\frac{\left|\zeta_{i}^{2}\right|}{R}$ unterhalb einer festen Schranke, woraus folgt, dass für ein noch grösseres $M$ sogar

$$
d n_{\mathrm{t}}>\frac{\left|\zeta_{i}^{2}\right| d \underline{g}}{M}
$$

gelten wird. Da $d n_{2}$ zu $d n_{1}$ proportional ist, so erfüllt $d n_{2}$ eine ähnliche Ungleichung.

Vergleicht man dann die auf die $\zeta_{i}^{2}$-Ebene bezogene Grösse $\frac{d n_{2}}{\left|\zeta_{i}^{2}\right|}$ mit der zur $\zeta_{i}^{9}$-Ebene gehörigen Grösse $\frac{d n_{1}}{\left|\zeta_{i}^{3}\right|}$, so sieht man fast in derselben Weise ein, dass das Verhältnis dieser Grössen oberhalb einer festen positiven Schranke liegt. In der Tat folgt dieses sofort aus den Gleichungen (I I), wenn man bedenkt, dass 
die nicht-euklidischen Differentiale $\frac{\left|d \zeta_{i}^{2}\right|_{z}}{\eta_{2}}$ und $\frac{\left|d \zeta_{s}^{-9}\right|_{1}}{\eta_{1}}$ in den betrachteten Ebenen einander gleich sind, und dass das Verhältnis der Grössen $\frac{\eta_{2}}{R}$ und ${ }_{R}^{\eta_{1}{ }^{1}}$ ein prositives Minimum hat. In dieser Weise fortsetzend erkennt man schliesslich, dass fiir ein gewisses $M$ die Ungleichung

$$
\operatorname{Min}\left(d n_{1}, d n_{2}\right)>\frac{\left|\begin{array}{c}
c_{i} \\
\varsigma_{i}
\end{array}\right| d \varrho}{\bar{M}}
$$

für alle $\zeta_{i}^{k}$-Ebenen richtig ist. Allerdings sind in der $\vartheta_{i}^{*}-$ Ebene $d n_{1}$ und $d n_{2}$ nicht mehr proportional; dafür kann aber auf $d n_{2}$ in der letzten Ebene genan dieselbe Überlegung angewandt werden wie auf $d n_{1}$ in der ersten Ebene.

Wählt man nun $\boldsymbol{M}$ in (9) gemäss der oben gestellten Forderung, so ist es klar, dass das Integral ( 10 ) kleiner sein wird als das Flächenintegral

$$
\iint\left|\begin{array}{l}
d s \\
\left.d l_{i}\right|_{i} ^{2}
\end{array}\right|^{2} d \omega
$$

ausgestreckt über das von den Kurven $I_{\varrho}^{i, k}$ gebildete Gebiet. Demn das Flächen-

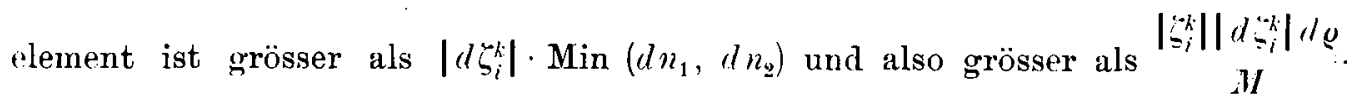

Dieses Integral stellt die Grösse der Bildfäche des genannten Gebiets dar. Führt man in (9) die Integration zwischen den Grenzen $\varrho^{\prime}$ und $\varrho^{\prime \prime}$ aus, so erhält man also auf der rechten Seite einen Ausdruck, der kleiner ist als der Gesamtinhalt einer Fläche, die einerseits von den Bildkurven der Kurven $I_{Q^{\prime}}^{\prime}$ und $I_{g^{\prime \prime}}^{\prime \prime}$, andererseits von den Geraden $\Im(s)=0$ und $\Im(s)=2 \pi$ begrenzt wird. Daher wird

$$
{ }_{q}^{4 \pi} \log \frac{\varrho^{\prime \prime}+K}{\rho^{\prime}+K}+\underset{q \pi}{\mathrm{I}} \int_{\varrho^{\prime}}^{\ddot{\prime}^{\prime \prime}} \frac{\omega(\varrho)^{2}}{\varrho+K} d \varrho \leqq 2 \pi \log \begin{aligned}
& r_{2}\left(\varrho^{\prime \prime}\right) \\
& r_{1}\left(\varrho^{\prime}\right)
\end{aligned}
$$

oder

$$
{ }_{q}^{2} \log \frac{\varrho^{\prime \prime}}{\varrho^{\prime}}-\log \frac{r_{1}\left(\varrho^{\prime \prime}\right)}{r_{1}\left(\varrho^{\prime}\right)} \leqq \omega\left(\varrho^{\prime \prime}\right)-{ }_{2 q \pi} \mathrm{I} \int_{\varrho^{\prime}}^{\varrho^{\prime \prime}} \frac{\omega(\varrho)^{2}}{\varrho+K} d \varrho+O\left(\begin{array}{c}
\mathrm{I} \\
\varrho^{\prime}
\end{array}\right)
$$

Diese Ungleichung entspricht der Ungleichung (2) in Nr. 4.

' $k$ hat in den heiden Fibenen verschiedene Bedeutung. 
Genau wie im Falle $q=3$ kann man aus dieser Ungleichung zunächst schliessen, dass $\lim _{\varrho \rightarrow \infty} v_{1}(\varrho)=\infty$ ist, d. h. dass das Existenzgebiet von $w(z)$ die ganze Ebene ist. Wir sind also berechtigt die Figur 6 als Figur in der $z$-Ebene aufzufassen. Es sei $\theta_{i}(r)$ der Zentriwinkel desjenigen auf dem Kreise $|z|=r$ gelegenen Bogens, der den linken Ufer von $C_{i}^{q-1}$ mit dem rechten Ufer von $C_{i+1}^{2}$ verbindet. Diese Bogen sind alle punktfremd und genügen folglich der Bedingung $\sum_{1}^{q} \theta_{i}(r) \leqq 2 \pi$.

Man beweist nun genau wie auf S. 385 die Ungleichungen

$$
\pi \int_{r_{2}\left(\varrho^{\prime}\right)}^{r_{1}\left(\varrho^{\prime \prime}\right)} \frac{d r}{r \theta_{i}(r)} \leqq \log \frac{\varrho^{\prime \prime}}{\varrho^{\prime}}+O\left(\frac{\mathrm{I}}{\varrho^{\prime}}\right), \quad(i==\mathrm{I}, \ldots, q) .
$$

Durch Addition folgt

$$
\pi \sum_{1}^{q} \int_{r_{2}\left(\varrho^{\prime}\right)}^{r_{1}\left(\varrho^{\prime \prime}\right)} \frac{d r}{r \bar{\theta}_{i}(r)} \leqq q \log \frac{\varrho^{\prime \prime}}{\varrho^{\prime}}+O\left(\frac{\mathrm{I}}{\varrho^{\prime}}\right)
$$

und, wenn man die Ungleichung

$$
\sum_{1}^{q} \frac{\mathrm{I}}{\theta_{i}(v)} \geqq \frac{q^{2}}{\sum_{1}^{q} \theta_{i}(v)} \geqq \frac{q^{2}}{2 \pi}
$$

benützt,

$$
\frac{q}{2} \log \frac{r_{1}\left(\varrho^{\prime \prime}\right)}{r_{2}\left(\varrho^{\prime}\right)} \leqq \log \frac{\varrho^{\prime \prime}}{\varrho^{\prime}}+o\left(\frac{\mathrm{I}}{\varrho^{\prime}}\right)
$$

oder

$$
{ }_{q}^{2} \log \frac{\varrho^{\prime \prime}}{\varrho^{\prime}}-\log \frac{r_{1}\left(\varrho^{\prime \prime}\right)}{r_{1}\left(\varrho^{\prime}\right)} \geqq-\omega\left(\varrho^{\prime}\right)+O\left(\frac{\mathrm{I}}{\varrho^{\prime}}\right)
$$

Zusammen mit $\left(2^{\prime}\right)$ ergibt sich also

$$
\omega\left(\varrho^{\prime}\right)+\omega\left(\varrho^{\prime \prime}\right) \geqq \frac{\mathrm{I}}{2 q \pi^{2}} \int_{\varrho^{\prime}}^{\rho^{\prime \prime}} \frac{\omega(\varrho)^{2}}{\varrho+\bar{K}} d \varrho+o\left(\frac{\mathrm{I}}{\varrho^{\prime}}\right)
$$

und aus dieser Ungleichung lässt sich wie in Nr. 6 schliessen, dass 51-31356. Acta mathematica. 58. Imprimé le 12 mars 1932. 


$$
\omega(\varrho)=O\left(\begin{array}{c}
\mathrm{I} \\
\sqrt{\log \varrho}
\end{array}\right)
$$

ist.

Aus den Ungleichungen (2') und $\left(5^{\prime}\right)$ folgt dann die asymptotische Darstellung

$$
\begin{aligned}
& r_{1}(\varrho)=A \varrho^{\frac{2}{a}}\left(\mathrm{I}+O\left(\frac{\mathrm{I}}{3} \sqrt{\log \varrho}\right)\right) \\
& r_{2}(\varrho)=A \varrho^{\frac{2}{q}}\left(\mathrm{I}+O\left(\frac{\mathrm{I}}{3}\right)\right)
\end{aligned}
$$

wo $A$ eine Konstante ist.

Die Anzahl $n(r, a)$ der im Kreise $|z|<r$ gelegenen $a$-Stellen der Funktion $w(z)$ erhält man nun sofort, wenn man beachtet, dass

$$
n\left(r_{1}(\varrho), a\right) \leqq n(\varrho, a) \leqq n\left(r_{2}(\varrho), a\right)
$$

ist, und die asymptotischen Darstellungen von $\nu(\varrho, a)$ S. 396 verwendet. Man findet so

$$
\begin{gathered}
n(r, a)=q A^{\prime} r^{\frac{q}{2}}\left(\mathrm{I}+O\left(\begin{array}{c}
\mathrm{I} \\
3 \\
\sqrt{\log r} i
\end{array}\right)\right) \text { für } a \neq a_{1}, \ldots, a_{t}, \\
n\left(r, a_{i}\right)=(q-2) A^{\prime} r^{\frac{q}{2}}\left(\mathrm{I}+O\left(\begin{array}{c}
\mathrm{I} \\
3 \\
\sqrt{\log r}
\end{array}\right)\right), \quad(i=\mathrm{I}, \ldots, q),
\end{gathered}
$$

wo $A^{\prime}=A^{-4}$.

Also ist auch

$$
n(r)=\operatorname{Max} n(r, a)=q A^{\prime} r^{\frac{q}{2}}\left(\mathbf{I}+O\left(\frac{\mathbf{I}}{\sqrt[3]{\log } r^{\prime}}\right)\right)
$$

d. h. $w(z)$ ist eine meromorphe Funktion vom mittleren Typus der Ordnung $\frac{q}{2}$. Ihre einzigen defekten Werte sind $a_{1}, a_{2}, \ldots, a_{q}$ mit den Defekten

$$
\delta\left(a_{i}\right)=\lim _{r \rightarrow \infty} \frac{n(r)-n\left(r, a_{i}\right)}{n(r)}=\frac{2}{q} .
$$

Die Defektsumme ist $q \cdot \frac{2}{q}=2$. 
B. I2. Wir haben dann noch den Fall zu erledigen, wo nicht alle Singularitäten ohne Umlauf der Punkte $a_{i}$ erreichbar sind. In diesem Falle kann es eintreffen, dass über demselben Punkt $a_{i}$ mehrere, etwa $\mu_{i}$, Singularitäten liegen. Die Summe $n=\sum_{1}^{q} \mu_{i}$ gibt die Gesamtanzahl der verschiedenen Singularitäten.

Die Struktur der Riemannschen Fläche $F$ geht nach Nevandinna aus einem Polygon mit $n$ Ecken hervor, von denen $\mu_{1}$ mit $a_{1}, \mu_{2}$ mit $a_{2}$ usw. bezeichnet sind. Dieses Grundpolygon ist noch durch einander nicht schneidende Diagonale in Teilpolygone von der Eigenschaft zerlegt, dass keine gleichbezeichnete Eckpunkte als Ecken desselben Teilpolygons auftreten. Jeder Diagonale wird

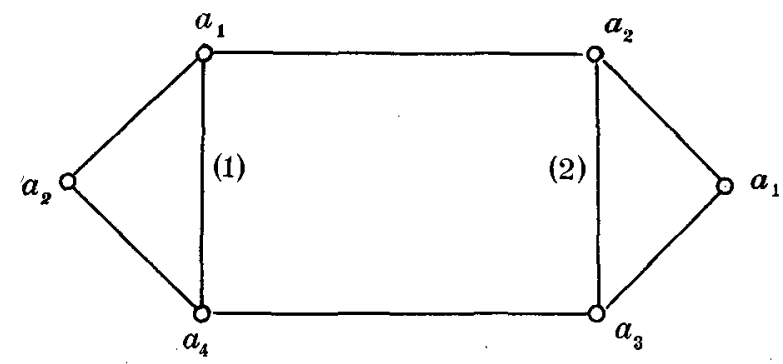

Fig. 9 ,

noch eine ganze Zahl zugeordnet; welche angibt, wie vielfach die betreffende Diagonale zu zählen ist. In Fig. 9 ist ein Schema für $\mu_{1}=\mu_{2}=2, \mu_{3}=\mu_{4}=$ I gezeichnet; die Diagonale $\left(a_{1}, a_{4}\right)$ wird einfach, die Diagonale $\left(a_{2}, a_{3}\right)$ doppelt gerechnet.

Die Bedeutung der schematischen Figur ist folgende: Jedem Teilpolygon entspricht ein Blatt der Riemannschen Fläche $F$, und in diesem Blatt sind diejenigen Punkte $a_{i}$ singulär, die unter den Ecken des Teilpolygons vorkommen. Dabei werden auch die $k$-fachen Diagonale als $k-\mathrm{I}$ aneinander gelegte Zweiecke aufgefasst, und geben mithin zu Blättern mit nur zwei Singularitäten Anlass. Die Blätter hängen in der von der schematischen Figur angegebenen Weise zusammen. Schliesslich werden noch an die Seiten des Grundpolygons 》logarithmische Enden» angehängt, die aus unendlich vielen, nur um die zwei zu den Endpunkten der betreffenden Seite gehörigen Punkte herumgewundenen Blättern bestehen.

Die zwei Singularitäten des $i$ :ten logarithmischen Endes seien $a_{i_{1}}$ und $a_{i_{q}}$; die übrigen Punkte bezeichnen wir in einer beliebigen Reihenfolge mit $a_{i_{2}}, a_{i_{3}}$, 
$\ldots, a_{i_{q-1}}$. Die Fläche $F$ wird genau wie in den vorhin behandelten Fällen längs Schnitten $C_{i}^{k}$ aufgeschnitten, die durch alle zu dem v:ten logarithmischen Ende gehörigen regulären Punkte über $a_{i_{k}}$ hindurchgehen. Die Kurve $C_{i}^{k}$ soll um die Punkte $a_{i_{1}}, a_{i_{2}}, \ldots, a_{i_{k-1}}$ im negativen und um die Punkte $a_{\mathrm{i}_{k+1}}, a_{i_{k+2}}$, $\ldots, a_{i q}$ im positiven Sinn herumlaufen. Die einzelnen Umläufe sollen genau übereinander liegen und in $\operatorname{der} \zeta=\zeta\left(w, a_{1}, \ldots, a_{q}\right)$-Ebene auf Orthogonalkreisbogen des Einheitskreises abgebildet werden.

Alsdann werden noch die regulären Punkte des Grundpolygons in willkürlicher Weise mit den Endpunkten der Kurven $C_{i}^{k}$ verbunden durch Kurvenstücke, die einander nicht schneiden dürfen, sonst aber völlig beliebig sind. Die endgültigen Schnitte, deren Anzahl $n(q-2)$ ist, bestehen also aus je einer der Kurven $C_{i}^{k}$ nebst einem angehängten Kurvenstück, das durch einen oder mehrere der Punkte $a_{v}$ des Grundpolygons hindurchgeht.

Auf der so aufgeschnittenen Fläche $F$ wird jeder Zweig von $\zeta(w)$ eindeutig. Dem Anfangspunkt des Schnittes $C_{i}^{k}$ entsprechen aber jetzt im allgemeinen zwei verschiedene Punkte auf der Peripherie des Einheitskreises, etwa $S_{i, k}^{\prime} \varepsilon_{i_{k}}$ und $S_{i, k}^{\prime \prime} \varepsilon_{i_{k}}$, wo $S_{i, k}^{\prime}$ und $S_{i, k}^{\prime \prime}$ gewisse Transformationen aus der Gruppe $S$ sind, in bezug auf welche die Funktion $\zeta(w)$ automorph ist. Da man auf der aufgeschnittenen Fläche vom Anfangspunkt des Schnittes $C_{i}^{2}$ zum Anfangspunkt von $C_{i-1}^{q-1}$ kommen kann, indem man nur den Punkt $a_{i_{1}}=a_{(i-1)_{q}}$ zweimal im positiven Sinn umläuft, so ist $S_{i-1, q-1}^{\prime \prime} \equiv S_{i, 2}^{\prime} S_{i_{1}}^{2}$. Die Anfangspunkte der Schnitte $C_{i}^{k}$ und $C_{i}^{k+1}(k=2, \ldots, q-2)$ können ohne irgendeinen Umlauf verbunden werden. Demnach ist $S_{i, k+1}^{\prime} \equiv S_{i, k}^{\prime \prime}$.

$\mathrm{Zu}$ den übrigen Punkten $a_{i_{k}}$ auf dem linken Ufer von $C_{i}^{k}$ gehören die Punkte $S_{i, k}^{\prime} T_{i, k}^{\prime \lambda} \varepsilon_{i_{k}}, \quad \lambda=\mathrm{I}, 2, \ldots \quad$ Hierbei ist $T_{i, k}^{\prime}=S_{i_{1}}^{-1} S_{i_{2}}^{-1} \ldots S_{i_{k-1}}^{-1}$. Zu den Punkten auf dem rechten Ufer gehören die Punkte $S_{i, k}^{\prime \prime} T_{i, k}^{\prime \prime \lambda} \varepsilon_{i_{k}}, \lambda=\mathrm{I}, 2, \ldots$, wo $T_{i, k}^{\prime \prime}=S_{i_{q}} S_{i_{q-1}} \ldots S_{i_{k+1}}$. Die Bilder der beiden Ufer erhält man, wenn man die zwischenliegenden Orthogonalkreisbogen zeichnet. Diese Kreisbogen häufen sich gegen die Punkte $S_{i, k}^{\prime} \omega_{i, k}^{\prime}$ und $S_{i, k}^{\prime \prime} \omega_{i, k}^{\prime \prime}$, wo $\omega_{i, k}^{\prime}$ und $\omega_{i, k}^{\prime \prime}$ die anziehenden Fixpunkte der Transformationen $T_{i, k}^{\prime}$ und $T_{i, k}^{\prime \prime}$ bezeichnen. Für $k=2, \ldots, q-2$ gilt wieder $\omega_{i, k}^{\prime \prime}=\omega_{i, k+1}^{\prime}$, denn $T_{i, k}^{\prime \prime} \equiv T_{i, k+1}^{\prime}$. Insgesamt gibt es $n$ parabolische und $n(q-3)$ hyperbolische Häufungspunkte, denn wegen der oben bewiesenen 
Relationen ist $S_{i, 2}^{\prime} \varepsilon_{i_{1}}=S_{i-1, q-1}^{\prime \prime} S_{i_{1}}^{-2} \varepsilon_{i_{1}}=S_{i-1, q-1}^{\prime \prime} \varepsilon_{i_{1}}=S_{i-1, q-1}^{\prime \prime} \varepsilon_{(i-1)_{q-1}}$ und $S_{i, k}^{\prime \prime} \omega_{i, k}^{\prime \prime}$ $=S_{i, k+1}^{\prime} \omega_{i, k}^{\prime \prime}=S_{i, k+1}^{\prime} \omega_{i, k+1}^{\prime}$.

Der Übergang zur $\zeta_{i}$-Ebene geschieht dann durch eine lineare Transformation, welche den Punkt $S_{i_{2}}^{\prime} \varepsilon_{i_{1}}=S_{i-1, q-1}^{\prime \prime} \varepsilon_{(i-1)_{q}}$ ins Unendliche wirft, und bei welcher der zugehörigen parabolischen Transformation eine Translation um eine Einheit entspricht. Die Rollen der Punkte $A$ und $B$ in Fig. 8 werden von den zul $S_{i, 2}^{\prime} \varepsilon_{i_{2}}$ und $S_{i-1, q-1}^{\prime \prime} \varepsilon_{(i-1)_{q-1}}$ gehörigen Bildpunkten übernommen. Alsdann kann man die Querschnitte $T_{\varrho}^{i}$ in derselben Weise definieren wie vorhin. Zur $\zeta_{i}^{k}$-Ebene kommt man durch eine Transformation, welche den Punkt $\omega_{i, k}^{\prime}$ in den Punkt $\infty$ überführt. Auch die Definitionen von $\Gamma_{\rho}^{i, k}$ können ohne weiteres übernommen werden.

Man kann nun denselben Beweis wie im vorigen Abschnitt durchführen. An die Stelle von $q$ kommt jetzt die Anzahl $n$ der parabolischen Fixpunkte, d. $h$. die Anzahl der verschiedenen logarithmischen Singularitäten. Als Endresultat bekommt man also die asymptotische Darstellung

$$
\begin{aligned}
& r_{1}(\varrho)=A \stackrel{\check{n}}{(}\left(I+O\left(\begin{array}{c}
I \\
3 \\
\sqrt{\log \varrho}
\end{array}\right)\right) \\
& r_{2}(\varrho)=A \varrho^{\frac{2}{n}}\left(I+O\left(\begin{array}{c}
I \\
3 \\
\sqrt{\log \varrho}
\end{array}\right)\right)
\end{aligned}
$$

Ébenso erhält man hieraus für $a \neq a_{1}, \ldots, a_{q}$

$$
n(r, a)=n A^{\prime} r^{n}\left(\mathrm{I}+O\left(\begin{array}{c}
\mathrm{I} \\
3 \sqrt{\log r}
\end{array}\right)\right)
$$

Die $a_{i}$-Stellen fehlen in allen logaritmischen Enden, deren eine Singularität gleich $a_{i}$ ist. Da die Anzahl dieser Enden $2 \mu_{i}$ ist, so folgt

$$
n\left(r, a_{i}\right)=\left(n-2 \mu_{i}\right) A^{\prime} r^{\frac{n}{2}}\left(\mathrm{I}+O\left(\frac{\mathrm{I}}{\sqrt[3]{\log r}}\right)\right) .
$$

Also ist

$$
n(r)=\operatorname{Max} n(r, a)=n A^{\prime} r^{\frac{n}{2}}\left(\mathrm{I}+O\left(\frac{\mathrm{I}}{\sqrt[3]{\log r}}\right)\right)
$$


und

$$
\delta\left(a_{i}\right)=\lim _{r \rightarrow \infty} \frac{n(r)-n\left(r, a_{i}\right)}{n(r)}=\frac{2 \mu_{i}}{n}
$$

Alle Funktionein $w(z)$, deren Umkehrfunktionen keine algebraische und nur eine endliche Anzahl $n$ von transzendenten Singularitäten besitzen, von denen $\mu_{1}$ über $a_{1}, \mu_{2}$ ïber $a_{2}$, usw. endlich $\mu_{q}$ über $a_{q}$ gelegen sind, sind in der ganzen z-Ebene meromorphe Funktionen rom mittleren Typus der Ordnung $\frac{n}{2}$, welehe nur die defekten. Werte $a_{1}, a_{2}, \ldots, a_{q}$ mit den Defekten

$$
\delta\left(a_{v}\right)=\frac{2 \mu_{v}}{n}
$$

besitzen. Die totale Defektsumme beträgt $\sum_{1}^{q} \frac{2 \mu_{v}}{n}=2$.

I3. Es verdient schliesslich hervorgehoben zu werden, dass das obige Resultat auch für solche Funktionen $w(z)$ gilt, deren Umkehrfunktionen ausser den $n$ transzendenten Singularitäten noch eine endliche Anzahl von algebraischen Singularitäten aufweisen. Um dieses zu beweisen müssen die Punkte, über welchen die algebraischen Verzweigungspunkte liegen, zu den. Punkten $a_{i}$ mitgerechnet werden, und bei der Aufschneidung der Riemannschen Fläche muss dafür gesorgt werden, dass die Schnitte auch durch alle algebraischen Singularitäten hindurchgehen. Da dieses auf die logarithmischen Enden keine Einwirkung hat, so wird man den Beweis genau in der obigen Weise durchführen.

Der bedeutungsvollste Punkt in der vorhergehenden Untersuchung war die Bestimmung des asymptotischen Verhaltens der fuchsoiden Funktion $z(\zeta)$ in der Umgebung der Ecken des Fundamentalgebiets. Die Anwendung auf die Wertverteilung der Funktion $w(z)$ ergab sich dann fast von sich selbst.

Unabhängig von dieser Anwendung besitzt die oben verwendete Methode auch eine allgemeine Bedeutung, welche darin liegt, dass sie die Bestimmung des asymptotischen Verhaltens einer fuchsschen oder fuchsoiden Funktion in der Umgebung einer Ecke ihres Fundamentalgebiets direkt aus dem Gestalt dieses Gebiets gestattet. Bekanntlich bietet diese Aufgabe bei Verwendung anderer Methoden erhebliche Schwierigkeiten. 